\title{
miRNA Profile in Hippocampus, Prefrontal Cortex and Sperm of Spontaneously Hypertensive Rats (SHR), a Model for Schizophrenia
}

\section{Gabriella Campagnoli}

Federal University of Sao Paulo

Isabelle Cantão

Federal University of Sao Paulo

Maryana Marins

Federal University of Sao Paulo

Agatha Mendes

Federal University of Sao Paulo

Beatriz Nogueira

Federal University of Sao Paulo

Renan Martin

Johns Hopkins University

João Pesquero

Federal University of Sao Paulo

Taiza Stumpp ( $\sim$ stumpp.taiza@unifesp.br)

Laboratory of Developmental Biology, Department of Morphology and Genetics - Escola Paulista de Medicina, Universidade Federal de São Paulo (UNIFESP), São Paulo, Brazil

\section{Research Article}

Keywords: schizophrenia, miRNAs, SHR, prefrontal cortex, hippocampus, sperm

Posted Date: December 9th, 2020

DOl: https://doi.org/10.21203/rs.3.rs-118724/v1

License: (c) (i) This work is licensed under a Creative Commons Attribution 4.0 International License.

Read Full License 


\section{Title Page}

miRNA Profile in hippocampus, prefrontal cortex and sperm of Spontaneously Hypertensive Rats (SHR), a model for Schizophrenia

Gabriella Mesas Campagnoli: Laboratory of Developmental Biology, Department of Morphology and Genetics - Escola Paulista de Medicina, Universidade Federal de São Paulo (UNIFESP), Sao Paulo, Brazil. Rua Botucatu, 740. Ed. Leitão da Cunha, 20 andar. CEP: 04023-900, São Paulo - SP, Brasil. Phone/Fax: 551155764262

Isabelle Hernandez Cantão: Laboratory of Developmental Biology, Department of Morphology and Genetics - Escola Paulista de Medicina, Universidade Federal de São Paulo (UNIFESP), Sao Paulo, Brazil. Rua Botucatu, 740. Ed. Leitão da Cunha, 20 andar. CEP: 04023-900, São Paulo - SP, Brasil. Phone/Fax: 551155764262

Maryana Mara Marins: Center for Research and Molecular Diagnosis of Genetic Diseases, Department of Biophysics - Escola Paulista de Medicina, Universidade Federal de São Paulo (UNIFESP), São Paulo, Brazil. Rua: Pedro de Toledo, 669. Ed. de Pesquisa II, $9^{\circ}$ andar, fundos. CEP: 04039-032, São Paulo - SP, Brasil. Phone: 55 1155764848.

Agatha Ribeiro Mendes: Center for Research and Molecular Diagnosis of Genetic Diseases, Department of Biophysics - Escola Paulista de Medicina, Universidade Federal de São Paulo (UNIFESP), São Paulo, Brazil. Rua: Pedro de Toledo, 669. Ed. de Pesquisa II, 9 andar, fundos. CEP: 04039-032, São Paulo - SP, Brasil. Phone: 55 1155764848. 
Beatriz Ribeiro Nogueira: Center for Research and Molecular Diagnosis of Genetic Diseases, Department of Biophysics - Escola Paulista de Medicina, Universidade Federal de São Paulo (UNIFESP), São Paulo, Brazil. Rua: Pedro de Toledo, 669. Ed. de Pesquisa II, 9 andar, fundos. CEP: 04039-032, São Paulo - SP, Brasil. Phone: 55 1155764848.

Renan Paulo Martin: McKusick-Nathans Department of Genetic Medicine, Johns Hopkins University School of Medicine, 733 N Broadway, Baltimore, MD 21205, USA

João Bosco Pesquero: Center for Research and Molecular Diagnosis of Genetic Diseases, Department of Biophysics - Escola Paulista de Medicina, Universidade Federal de São Paulo (UNIFESP), São Paulo, Brazil. Rua: Pedro de Toledo, 669. Ed. de Pesquisa II, $9^{\circ}$ andar, fundos. CEP: 04039-032, São Paulo - SP, Brasil. Phone: 55 1155764848.

Taiza Stumpp*: Laboratory of Developmental Biology, Department of Morphology and Genetics - Escola Paulista de Medicina, Universidade Federal de São Paulo (UNIFESP), São Paulo, Brazil. Corresponding author: stumpp.taiza@unifesp.br Rua Botucatu, 740. Ed. Leitão da Cunha, 2o andar. CEP: 04023-900, São Paulo - SP, Brasil. Phone/Fax: 551155764262

\section{ABSTRACT}

Environment is crucial for manifestation and development of schizophrenia, bringing to light epigenetic mechanisms involved, such as post-transcriptional control of gene expression by miRNA, in this disorder. MiRNAs participate in physiological processes such as neurogenesis, behavioral manifestations as well as in adverse conditions like psychiatric disorders. Experimental models are essential to investigate the development of schizophrenia from early life, especially its molecular aspects. 
Spontaneously hypertensive rats (SHR) are considered a good model for schizophrenia, since they show behavior that can be related to SCZ symptoms in humans and respond to treatment with anti-psychotic drugs. We investigated miRNA profile in prefrontal cortex, hippocampus and sperm of SHR through next generation sequencing (NGS). SHR showed differential expression (DE) of miRNAs related to neurological processes and schizophrenia in sperm, prefrontal cortex and hippocampus. Gene ontology and enrichment analyses showed that the majority of DE miRNA target genes are involved in pathways related to behavior, neurodevelopment and synaptic processes. Among these genes, 12 are dysregulated in schizophrenia patients, what reinforces SHR as a good model for epigenetic and molecular studies of this disorder. Finally, altered miRNA expression in sperm suggests that SHR model may be useful for future studies on epigenetic inheritance of schizophrenia.

Keywords: schizophrenia, miRNAs, SHR, prefrontal cortex, hippocampus, sperm.

\section{INTRODUCTION}

MicroRNA (miRNA) are small non-coding RNA molecules that interact with mRNA to control post-transcriptional gene expression. They function in a vast variety of fundamental biological processes, and many of them have been shown to be very conserved across animal species. Alterations of miRNA levels have been associated with different adverse conditions, including psychiatric disorders ${ }^{1-6}$. In mammals, approximately $70 \%$ of all miRNA described in the literature are expressed in the brain $^{7}$, indicating that they are particularly important for neurophysiology and neurodevelopmental processes.

Schizophrenia (SCZ) is a severe psychiatric condition that affects around $1 \%$ of the world population. People with this disorder has issues related to reality interpretation, to behavior, and to relationships that considerably impair their lives. Despite the considerable amount of studies about this disorder, its etiopathology is still 
obscure. There is a substantial amount of studies dedicated to investigate the genetic origins of SCZ and they have been providing relevant data on candidate genes and single nucleotide polymorphisms (SNP).

Although SCZ manifestation generally occurs in the adulthood, its origin is associated to the prenatal life. Therefore, the establishment of animal models is important to enable the investigation of the course of schizophrenia development. The Spontaneously Hypertensive Rat (SHR) is an inbred spontaneous model created at Okamoto Medical School, in 1963. Noteworthy, no significant genetic alteration in these rats has been detected ${ }^{8,9}$. Although these rats were originally described as a hypertension model, behavioral abnormalities, such as social and attention deficit, impulsivity, hyperactivity and hyperlocomotion have also been reported as important characteristics $^{10}-12$. Increased locomotor activity and reduced social interaction observed in SHR can be related to symptoms of SCZ in humans, such as social withdrawal and disorganized communication ${ }^{13-15}$. In behavioral and pharmacological studies using the SHR, these manifestations are reduced by the administration of antipsychotic drugs used to treat SCZ, such as clozapine ${ }^{16}$ and sodium nitroprusside ${ }^{17}$, reinforcing that this model can be used for SCZ studies. Although pharmacological and behavioral characteristics have been addressed in SHR model, to our knowledge studies on the molecular characteristics underlying the schizophrenia-like symptoms have not been studied.

Recent studies reported miRNAs are dysregulated in prefrontal cortex ${ }^{18}{ }^{19}$, in the hippocampus ${ }^{18}$, and in the peripheral blood ${ }^{20}$ of SCZ patients. Thus, the role of these molecules as possible SCZ markers or as targets for SCZ treatment has been considered. miRNAs are also part of the epigenetic mechanisms involved in diverse biological phenomena and responses to environmental influences. Although the genetic basis of SCZ has been largely studied and the evidences show it plays indeed a crucial role in SCZ etiology and inheritance, environmental factors are very important 
for its development and manifestation. Trauma and stress for example, have been pointed as catalysts of SCZ ${ }^{21-24}$.

As previously mentioned, the description of animal models to study developmental and molecular aspects of SCZ, which cannot be easily addressed in humans, is of great value to understand this disorder. The SHR have been pointed as a useful model to study behavioral and pharmacological aspects of SCZ. In addition, the importance of comprehending its inheritance and, more recently, its epigenetic characteristics reinforces the benefits of animal models to enable long term and extensive studies. Therefore, in the present study, we performed a global miRNA profile analysis of hippocampus, prefrontal cortex and sperm samples from SHR through next generation sequencing (NGS) using well established databases, performing functional annotations for both rats and humans.

\section{RESULTS}

Differentially expressed (DE) miRNAs in hippocampus, prefrontal cortex and sperm

Bioinformatics analysis of HIPPO, PFC and SPERM from Wistar and SHR resulted in the identification of 629 miRNAs. From these 629 miRNAs, 45 miRNAs were differentially expressed (DE) in the SPERM (Suppl. Fig. 1), 5 in the PFC (Suppl. Fig. 2), and 13 (Suppl. Fig. 3) in the HIPPO. Upregulated and downregulated miRNAs observed in the three samples are indicated in Table 1. From the DE miRNAs, miR130a was downregulated in sperm and PFC (Table 1). 


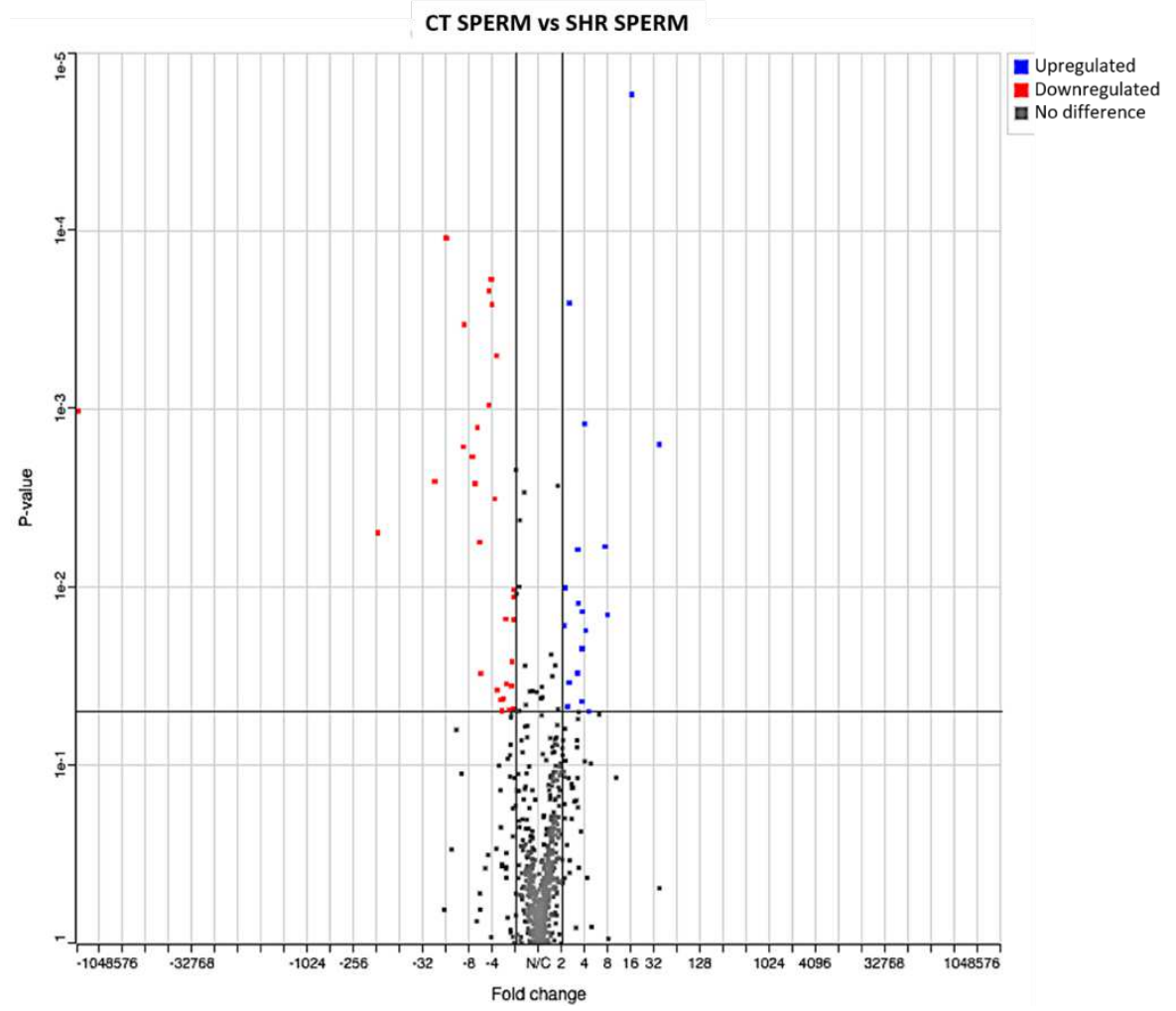

Suppl. Fig. 1: Volcano Plot: miRNAs expression in SPERM. Dots above the black horizontal line indicate the miRNAs whose expression analysis showed a value of $p$ $<0.05$. Gray dots represent miRNAs that showed no significant difference in expression between the two groups. The red and blue dots represent hypoexpressed and hyperexpressed miRNAs, respectively. Vertical lines: Fold change of \pm 2 . 


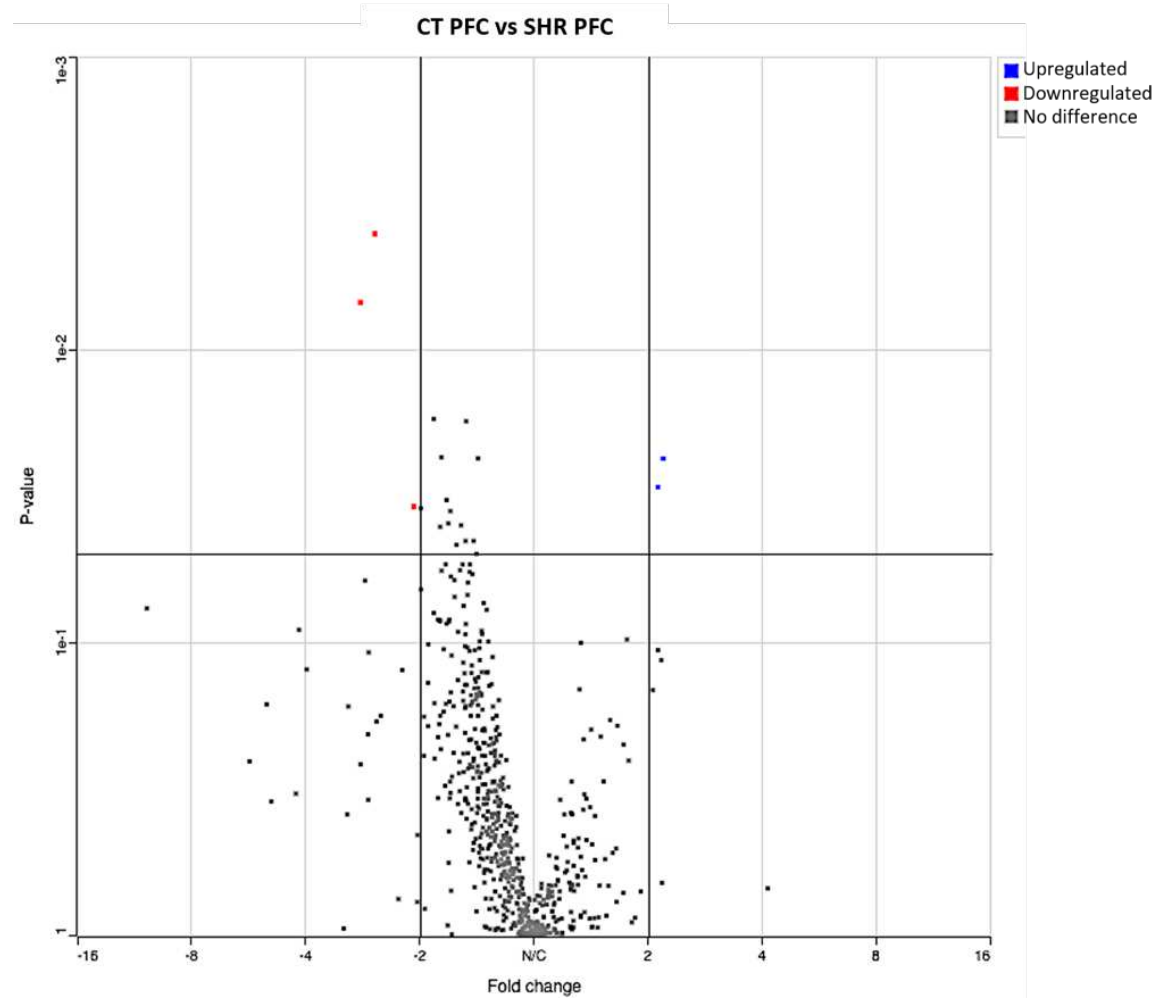

Suppl. Fig. 2: Volcano Plot: miRNAs expression for CPF. Dots located above the black horizontal line indicate the miRNAs whose expression analysis showed a value of $p$ $<0.05$. The gray dots represent miRNAs that showed no significant difference in expression between the two groups. The red and blue dots represent hypoexpressed and hyperexpressed miRNAs, respectively. Vertical lines: Vertical lines: Fold change of \pm 2 . 


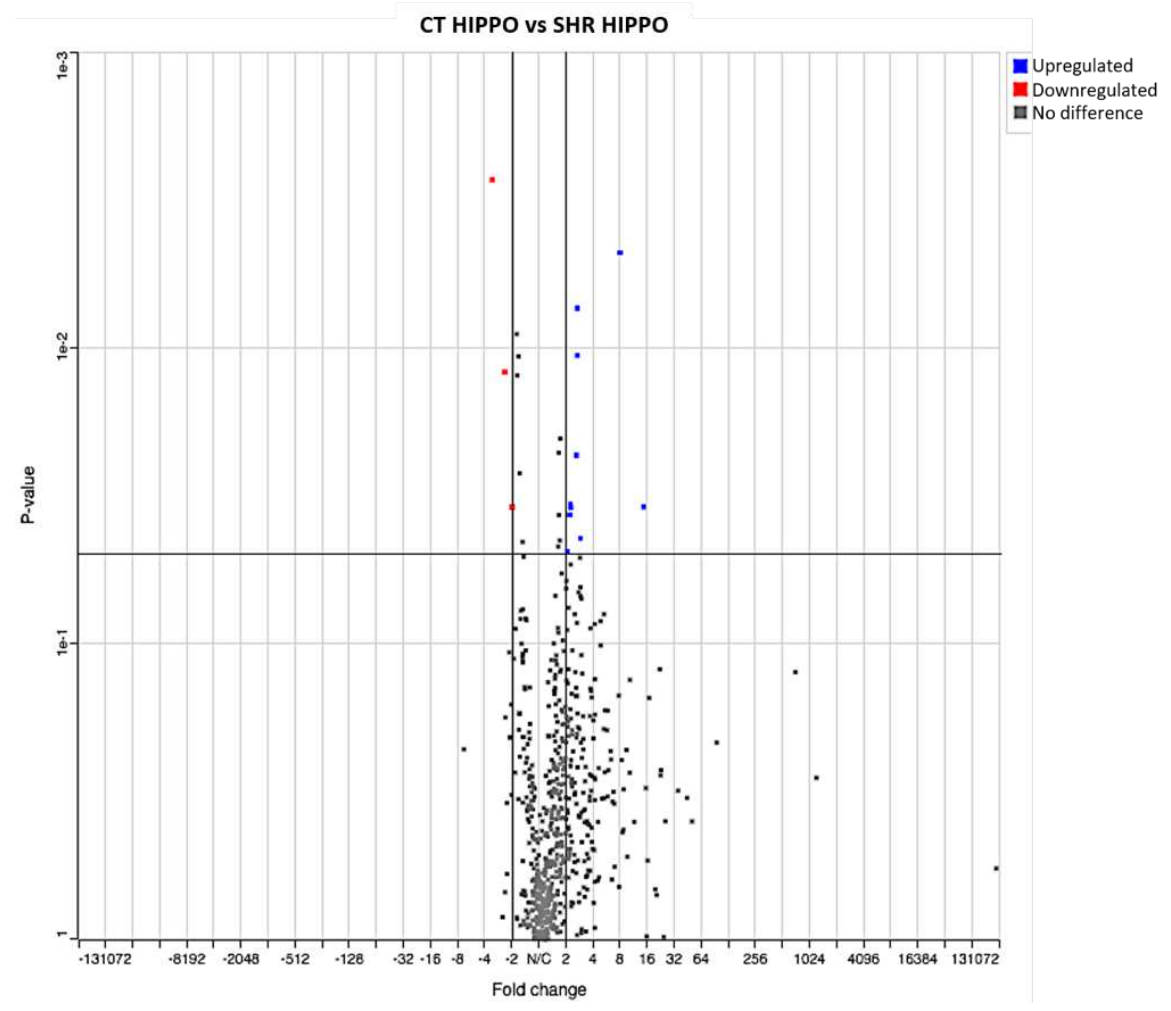

Suppl. Fig. 3: Volcano Plot: miRNAs expression in HIPO. Dots located above the black horizontal line indicate the miRNAs whose expression analysis showed a value of $p$ $<0.05$. Gray dots represent miRNAs that showed no significant difference in expression between the two groups. The red and blue dots represent hypoexpressed and hyperexpressed miRNAs, respectively. Vertical lines: Vertical lines: Fold change of \pm 2 . 
Table 1: Differentially expressed miRNA in SPERM, PFC and HIPPO of SHR when compared to control (Wistar) rats.

\begin{tabular}{|c|c|c|c|c|c|}
\hline \multicolumn{6}{|c|}{ Differentially Expressed miRNAs } \\
\hline \multicolumn{2}{|c|}{ SPERM } & \multicolumn{2}{|c|}{ PFC } & \multicolumn{2}{|c|}{ HIPPO } \\
\hline Downregulated & Upregulated & Downregulated & Upregulated & Downregulated & Upregulated \\
\hline $130 a$ & 210 & $130 a$ & 465 & 667 & 3546 \\
\hline $196 b-1$ & 541 & $31 a$ & 451 & 664 & $30 \mathrm{a}$ \\
\hline 93 & 365 & $551 b$ & & 150 & $344 b-1$ \\
\hline 223 & $434-2$ & & & & $17-1$ \\
\hline 33 & 423 & & & & 3589 \\
\hline let-7b & $148 b$ & & & & $26 b$ \\
\hline $34 a$ & 327 & & & & 499 \\
\hline $376 a$ & $1843 b$ & & & & 3562 \\
\hline $218 a$ & 1 & & & & $15 b$ \\
\hline 142 & 185 & & & & 16 \\
\hline $449 c$ & 877 & & & & \\
\hline 339 & let-7f-1 & & & & \\
\hline 320 & 377 & & & & \\
\hline 3558 & 6320 & & & & \\
\hline $106 b$ & 1188 & & & & \\
\hline 3543 & 671 & & & & \\
\hline 434 & 341 & & & & \\
\hline 375 & 544 & & & & \\
\hline 3072 & & & & & \\
\hline 423 & & & & & \\
\hline 1249 & & & & & \\
\hline 675 & & & & & \\
\hline 674 & & & & & \\
\hline 365 & & & & & \\
\hline let-7e & & & & & \\
\hline $322-2$ & & & & & \\
\hline 503 & & & & & \\
\hline $322-1$ & & & & & \\
\hline 708 & & & & & \\
\hline
\end{tabular}




\section{INTERACTIVE ANALYSIS OF DIFFERENTIALLY EXPRESSED miRNAs Differentially expressed miRNA target genes}

To investigate the possible consequences of changes in expression of the DE miRNAs in SPERM, PFC, and HIPPO, target (miRDB and TargeScan) and pathway interaction (Metascape) prediction tools were used. Hundreds of predicted target genes for the miRNAs differentially expressed in each sample were provided by TargetScan and miRDB platforms, generating more than 9000 target genes in the PFC, more than 25000 in the HIPPO and more than 75000 in the SPERM, what impair the subsequent bioinformatics analyses and graphical representations. Thus, to proceed with the interactive analyses, only the target genes related to nervous system processes were considered. Small differences between the results produced by TargetScan and miRDB platforms were observed; then, only the target genes that appeared in both databases were considered.

The relevant target genes predicted for the differentially expressed miRNAs in SPERM, PFC and HIPPO are represented by Cytoscape ${ }^{\circledR}$ networks (Fig. 1). These networks also show genes that are targeted by multiple miRNAs.

Because the number of differentially expressed miRNAs was higher in SPERM (Fig. 1A - C) than in PFC (Fig. 1D) and HIPPO (Fig. 1E), a considerably greater number of target genes were found in these cells. Thus, the network representation produced by Cytoscape was separated in three parts (Fig. 1A - C) to facilitate the illustration of the data. This separation was based in the number of target genes provided for each miRNA. 
$1 \mathrm{~A}$

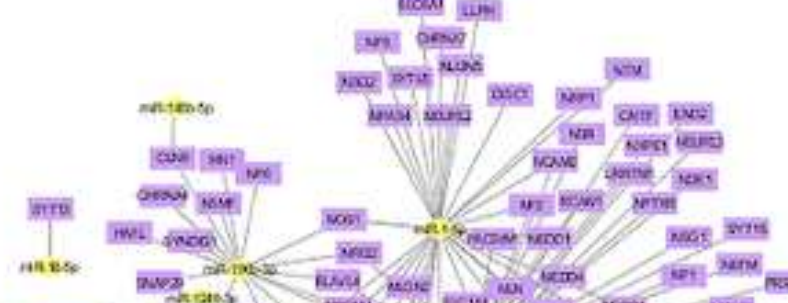

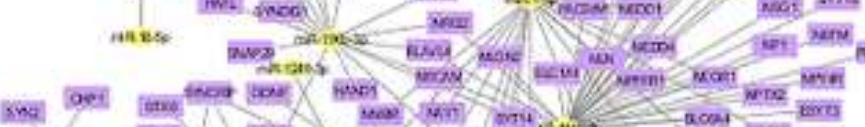

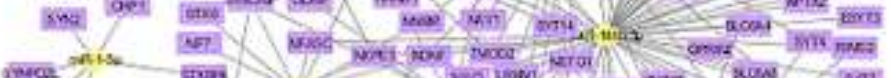

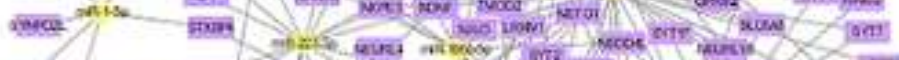

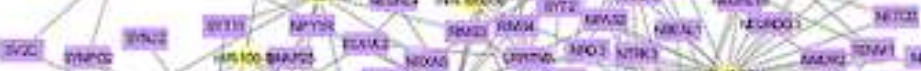
(1)

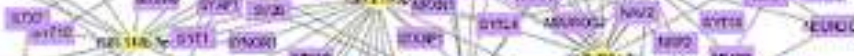

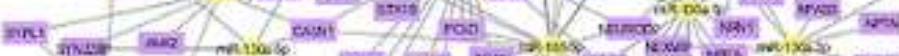

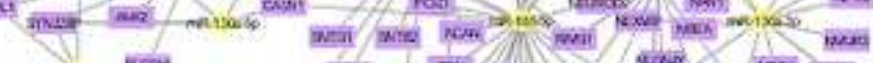

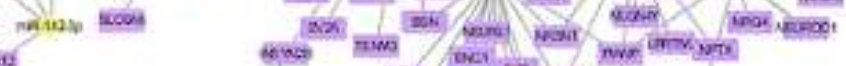
max

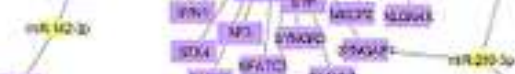
sout

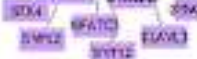

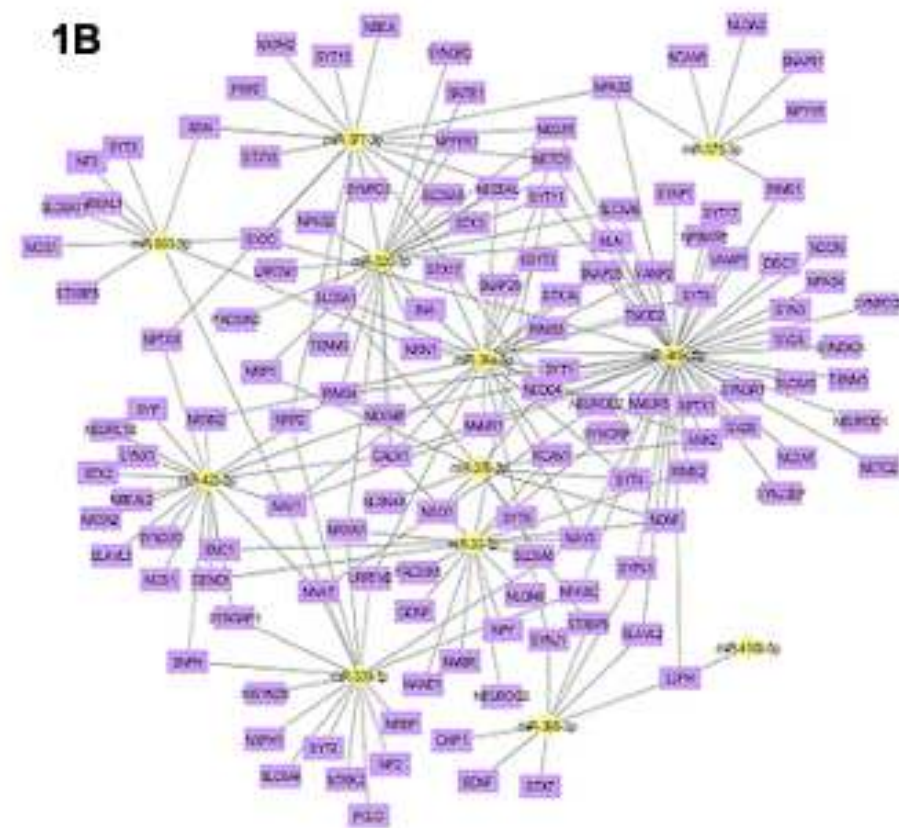

\section{$1 \mathrm{C}$}

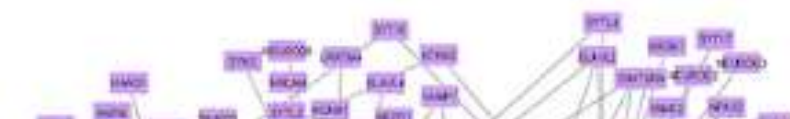


1D

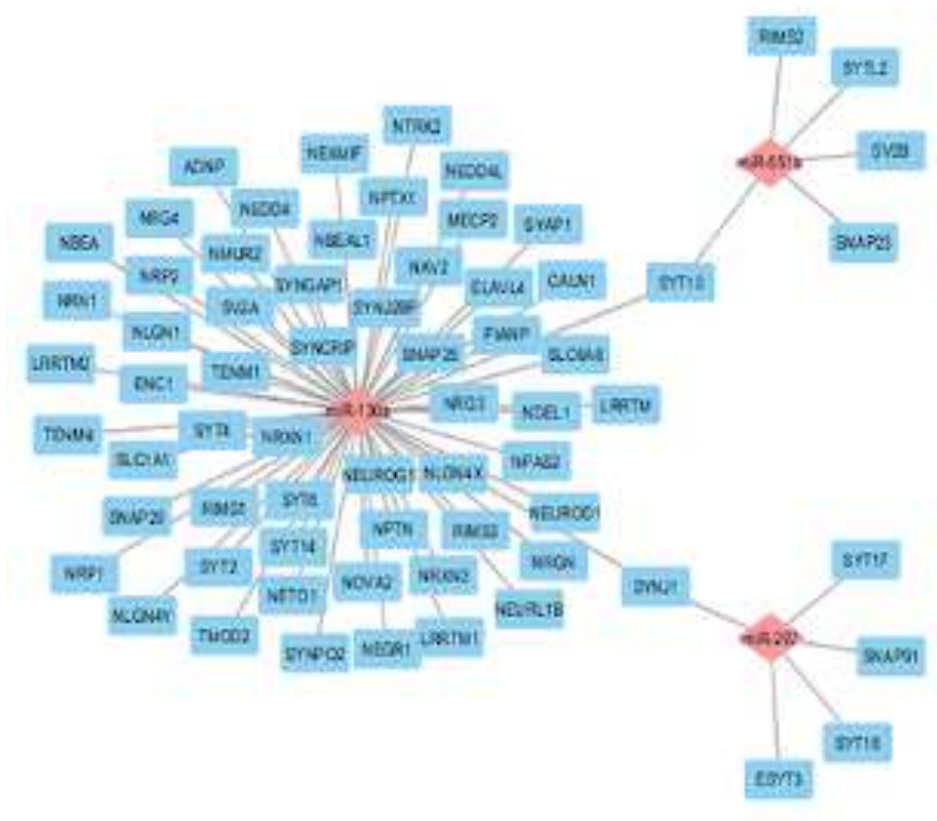

$1 \mathrm{E}$

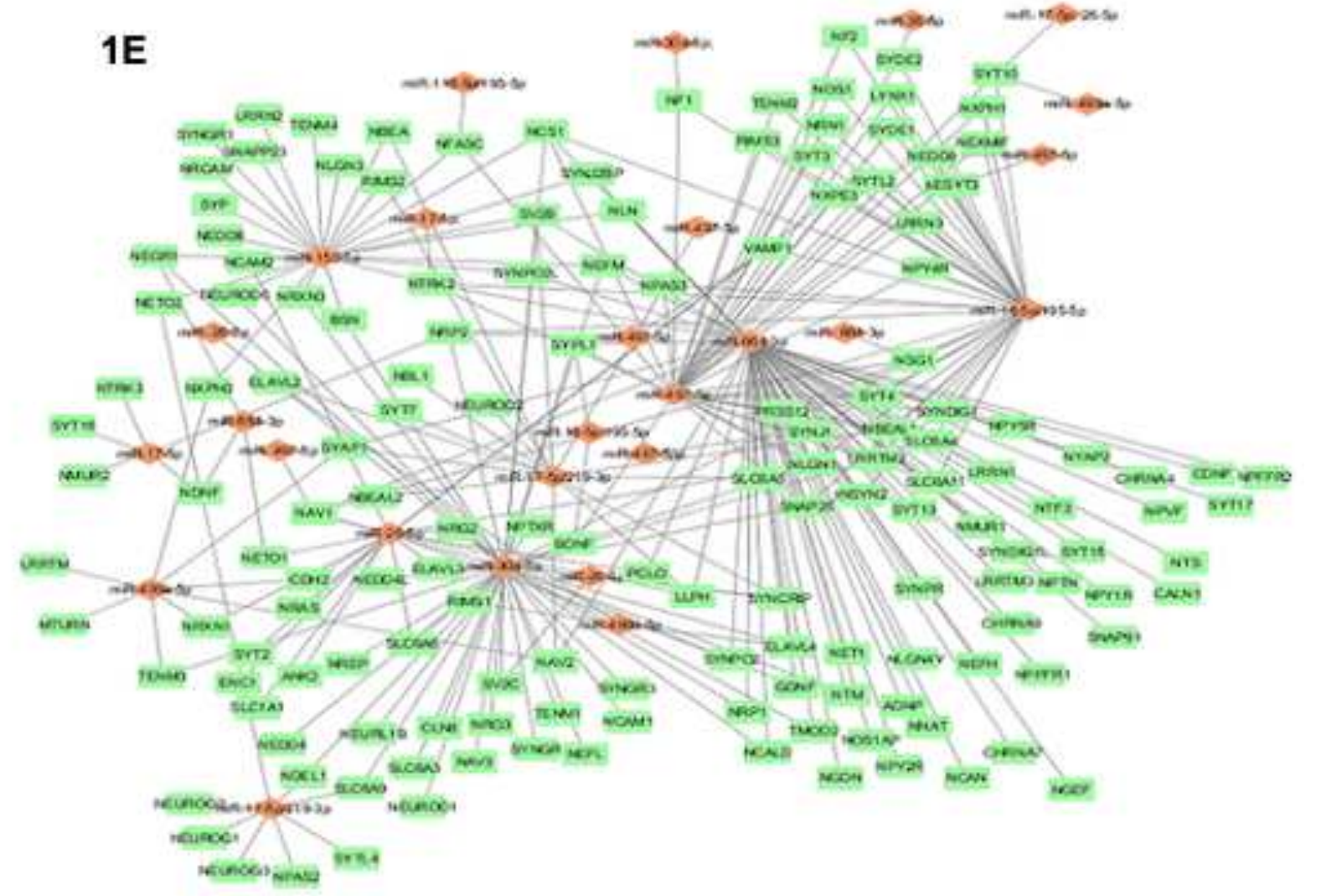

Fig. 1: Interaction map of the DE miRNAs (diamonds) and their target genes (rectangles) - in SPERM, part 1 (A), 2 (B) and 3 (C); CPF (D) and HIPPO (E). 
Ontology of miRNA target genes and enriched pathways

The analysis of the target genes of the DE miRNAs using the Metascape platform produced heatmaps of the enriched pathways in SPERM (Fig. 2A), CPF (Fig. 2B), and HIPPO (Fig. 2C). In the three types of samples, the top 20 enriched pathways indicated by Metascape include those related to synaptic processes, behavior, and neurodevelopment. 
2A

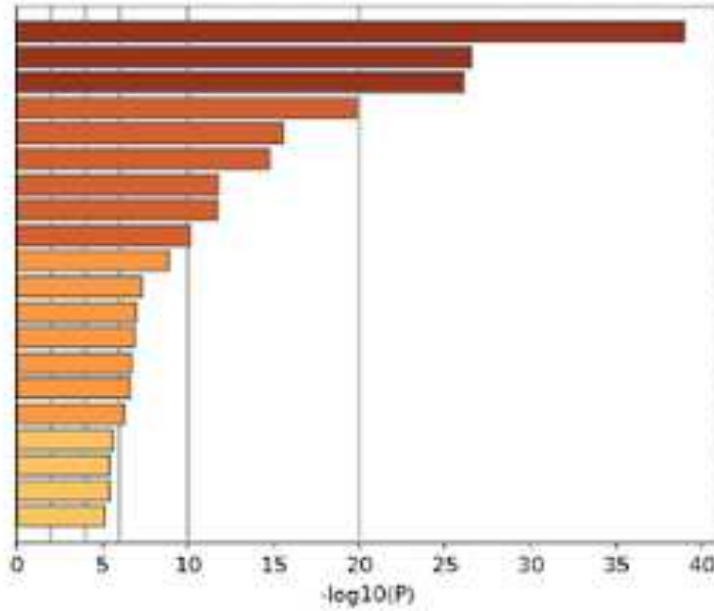

60.0006a36: neurotransmitter transport

60-0050804: modulation of chemical synaptic transmission

G0.0050808: synapse orgonization

60-0051962: positive reguiation of nervous system development

00.0022406 : merrbrane docking

G0:0051668: localization within mentrane

G0.0007612: lesrning or memery

60-0031644: regulation of neurologizel system process

60.0097091: synaptic vesicle clustering

G0.0036465: synaptic vesicle recycling

60.0001764: neuron migrotion

G0-0098917: retrograde trans-synaptic signaling

G0-0046879: homone secretion

Go:0066879: homone secretion

60.0050772 postive reguiotion of axe

0.0016358: dendrito devolopment

G0-0099540; trans-synaptic signaling by neurcpeptide

50:0001504: neurotransmitter uptake

G0.0032409: regulation of trensporter activity

60:004B168: regulatien of naurenal synaptic plastieity

G0:0090128: regulation of synapse maturation

\section{B}

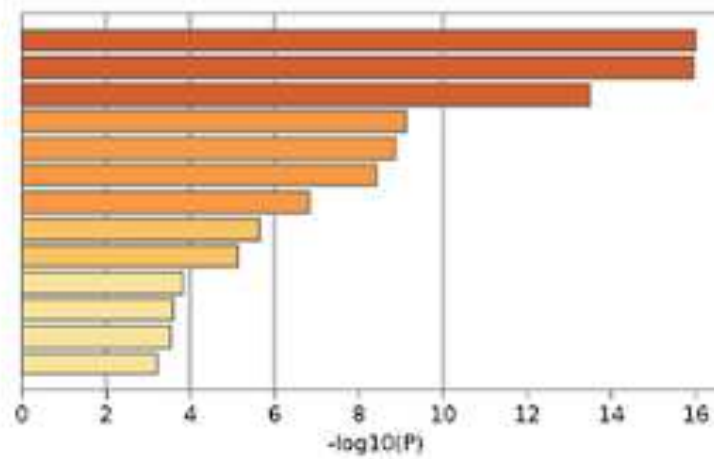

60:0007269 neurotransmitter secretion

60:0051962 pos tive regulation of nervous system development

60:0050804: modulation of chamical synaptic trensmission

GO:0140029: exocytic process

60:0007610: behavior

GO 0051668 : locelization within membrane

60.0030100 : regulation of endocytosis

60.0016358 dendrito dovelopment

60:0021783 prepanglionic porosympathetic fiber dovelepment

60.0007626 : locemotory behovior

60:0035176: social behavior

G0:0007613: memory

G0:0050768 negative regulation ef neuregenesis

2C

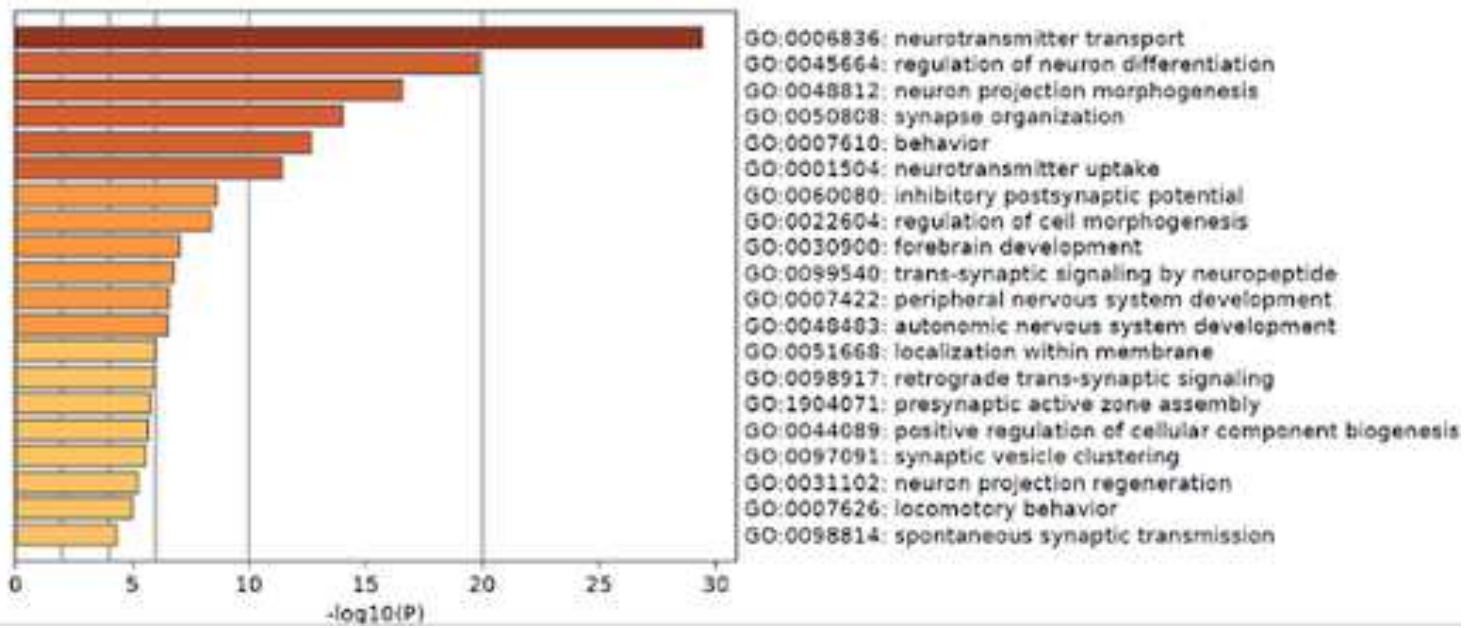

Fig. 2: Heatmap representation of the biological phenomena in which the DE miRNA in SPERM (A), PFC (B) and HIPPO (C) are involved. The colors of the bars indicate the values of $P$; the darker the colors, the lower the $\mathrm{P}$ value. Analysis was performed using rat database. 
Metascape analysis also provided the representation of the gene ontology of the target genes in the previously mentioned enriched pathways (Fig. 3). The data showed that SPERM (blue fraction) accounts for a large proportion of most enriched pathways (Fig. 3A). It was also observed that the pathways related to synapsis organization (Fig. 3B, green) and nervous system development (Fig. 3B, purple), which are considered to be important for SCZ pathogenesis, are interconnected and concentrate many target genes, as indicated by the size of the nodes. Other processes known to be involved in SCZ such as learning and memory (Fig. 3B, brown) also include a large amount of DE miRNA target genes.

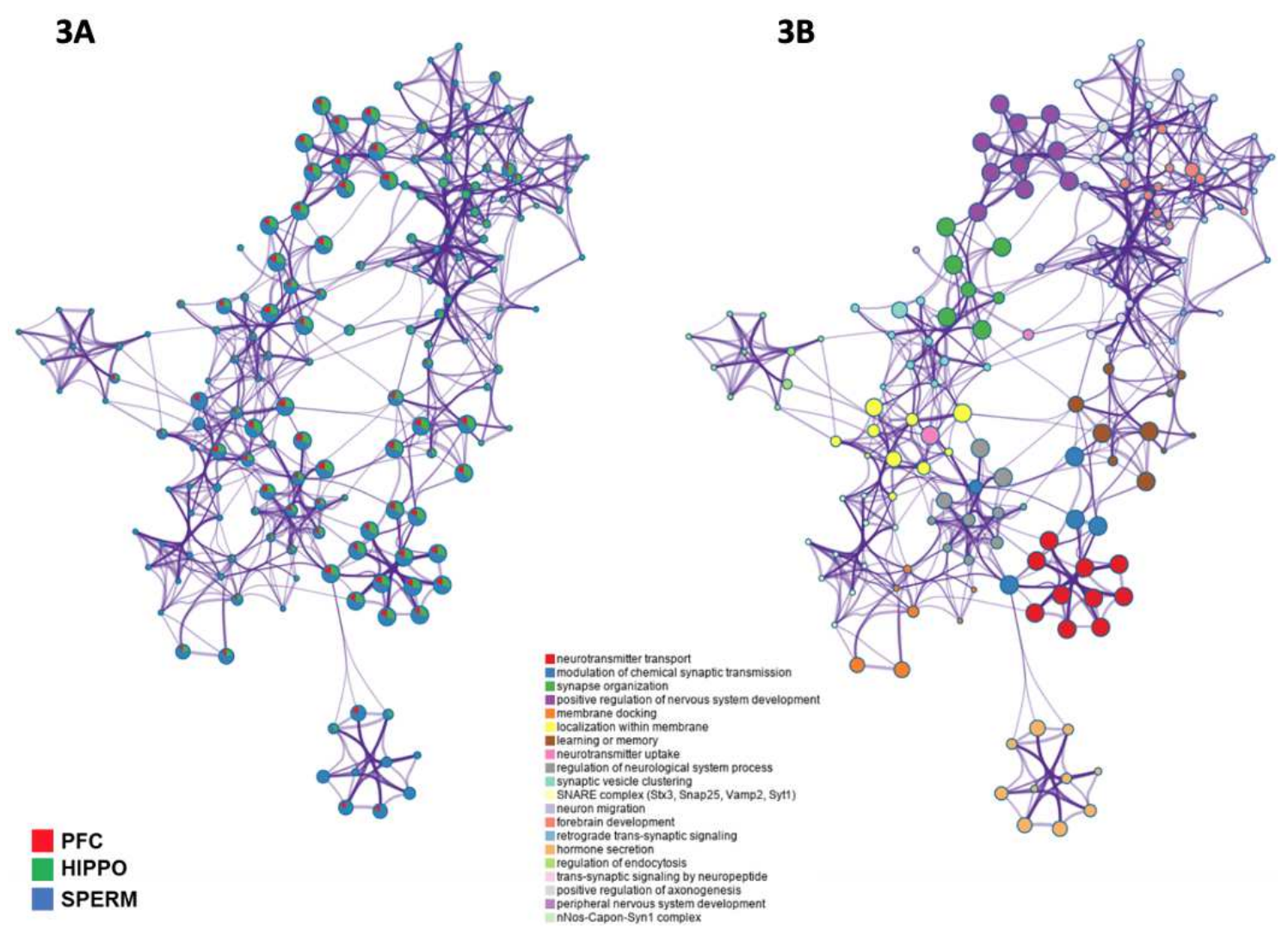

Fig. 3: Enrichment network (Gene Ontology Enrichment) in which the nodes represent the hits involved in each biological process. In A the nodes are represented as pie charts. Each portion of the pie represents a sample, and the size of each portion represents the proportion of each hit in each sample. Each hit includes the genes involved in biological processes, which are identified by the colors in $\mathrm{B}$. The size of the nodes reproduces the amount of genes involved in the hits and the purple lines between the nodes represent the interactions. Analysis using the rat database. 
A hierarchical heatmap was obtained (Fig. 4), where biological processes were related to each other and between the three types of sample. This data illustrates the connection between processes and show which ones are relevant in the SPERM, PFC and HIPPO.

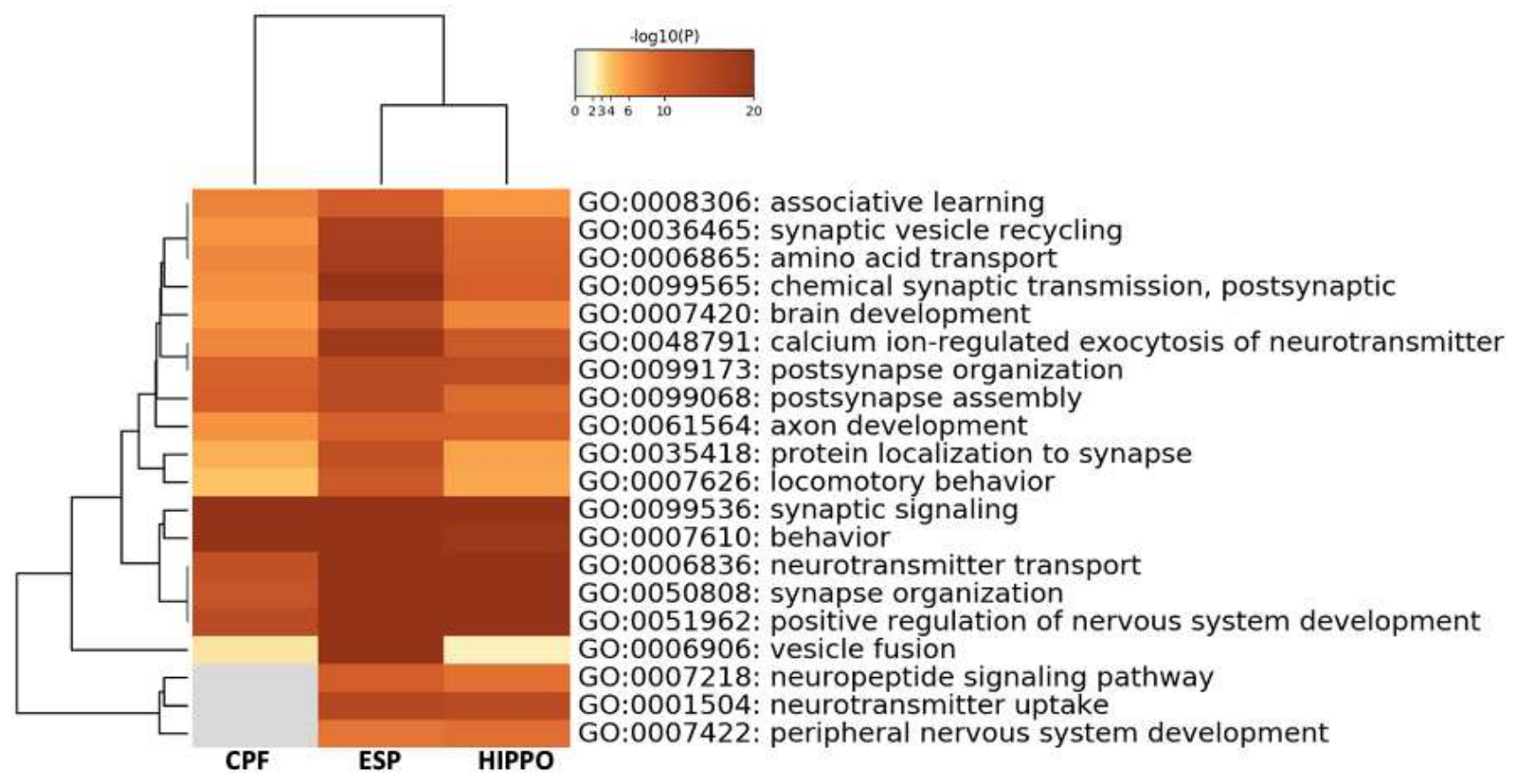

Fig. 4: Hierarchical heatmap illustrating the relationship between the identified biological processes and between three types of sample. The colors of the rectangles indicate the $P$ values; the darker the colors, the lower the value of $P$. It is observed that there is considerable similarity between the hippocampus and sperm. Synaptic organization and signaling, learning and memory, and neurodevelopment appear as processes that could be significantly affected in the three types of sample. Analysis was performed using the rat database. 
The Circus Plot (Fig. 5) represents the target genes that coincide between the three types of sample and those that appear only one type of sample.
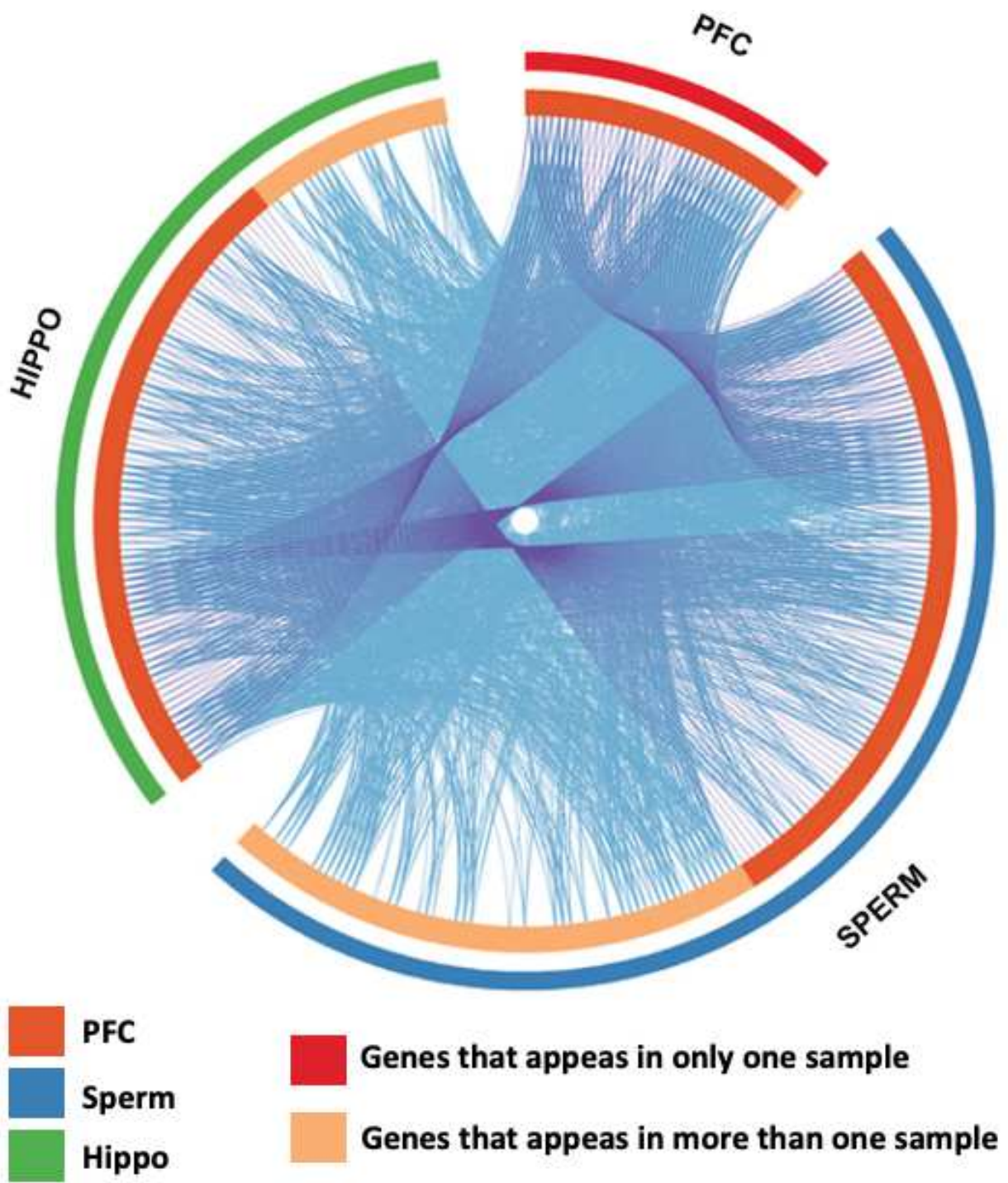

\section{Genes that appeas in only one sample}

\section{Genes that appeas in more than one sample}

Fig. 5: Circos Plot illustrates how differently expressed miRNA target genes coincide between the three types of sample. The outermost arches, represented in blue, red, and green, indicate sperm, prefrontal cortex, and hippocampus, respectively. The internal arcs, represented by the colors dark orange and light orange, represent the genes that appear in more than one type of sample or in a single type of sample, respectively. The purple lines indicate genes that appear in more than one type of sample. The blue lines link genes that are in the same "gene ontology term"; in different types of samples. Analysis was performed using rat database. 


\section{Translational Analysis}

Since SCZ is a disorder restricted to humans, and because one of the aims of this study was to corroborate the usefulness of the SHR strain as a model for molecular studies about this disorder, we also analyzed the DE miRNA using human databases available in TargetScan, miRDB and Metascape. These data are presented in supplementary figures 4 to 9 and are very similar to those obtained using the rat database. Interestingly, even more predicted target genes resulted from the TargetScan and miRDB analyses in the human database than in the rat database.

Subsequently, we consulted the SZDB database and found that, from the DE miRNA target genes, 12 show altered expression in SCZ patients. These genes are ADNP, LYNX1, NBEAL, NF1, NLGN1, NRSN1, SLC6A11, STX6, SYT2, SYT14, TENM1 and TENM3. Interestingly, from the 12 SCZ-related genes, 11 are targets for miRNAs differentially expressed in sperm (ADNP, LYNX1, NBEAL, NF1, NLGN1, NRSN1, SLC6A11, STX6, SYT14, TENM1 and TENM3); 5 are targets for miRNAs differentially expressed in PFC (ADNP, NBEAL1, NLGN1, SYT14 and TENM1); 9 are targets for miRNAs differentially expressed in HIPPO (ADNP, LYNX1, NBEAL1, NF1, NLGN1, SLC6A11, SYT2, TENM1 and TENM3). 


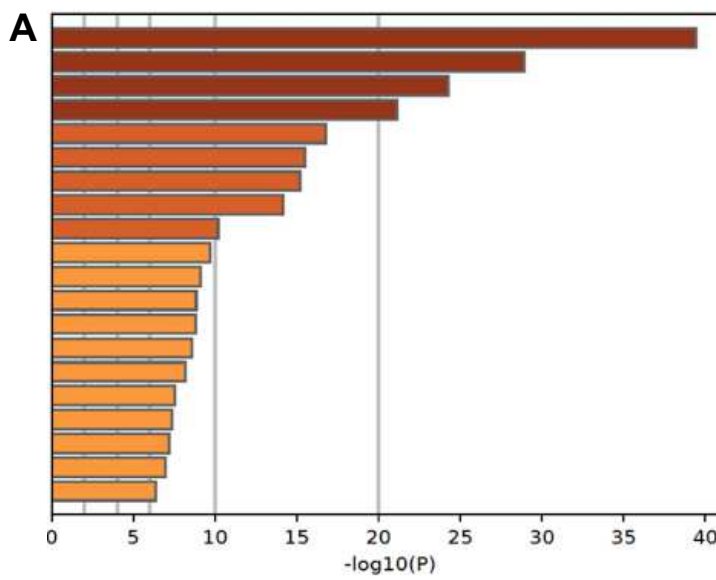

GO:0099537: trans-synaptic signaling

GO:0006836: neurotransmitter transport

GO:0051962: positive regulation of nervous system development

GO:0050808: synapse organization

GO:0048167: regulation of synaptic plasticity

R-HSA-112316: Neuronal System

GO:0001504: neurotransmitter uptake

GO:0042391: regulation of membrane potential

GO:0006865: amino acid transport

GO:0099068: postsynapse assembly

G0:0007422: postsynapse assembly

GO:0007218: neuropeptide signaling pathway

RO:0007218: neuropeptide signaling pathway

GO:0001764: neuron migration

RO:HSA-112315: Transmission across Chemical Synapse

R-HSA-112315: Transmission across Chemical Synapses

GO:0007420: brain development

GO:0060052: neurofilament cytoskeleton organization

GO:0050805: negative regulation of synaptic transmission

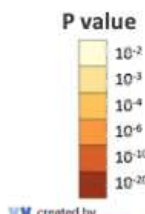

0

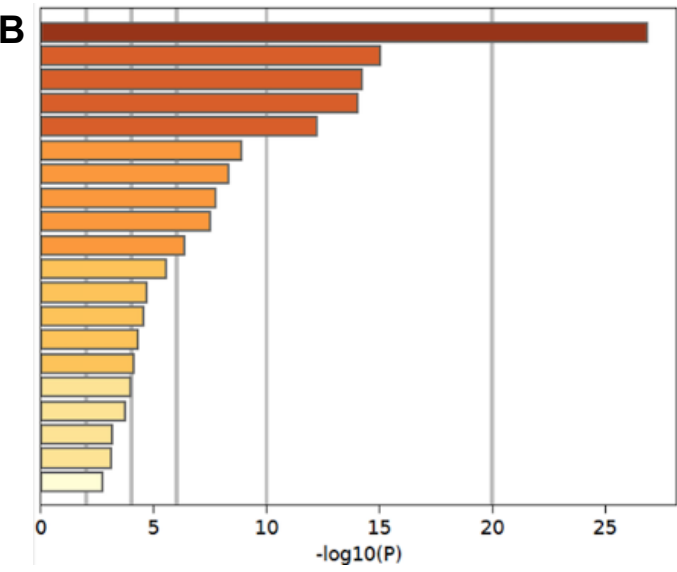

GO:0099536: synaptic signaling

GO:0099504: synaptic vesicle cycle

GO:0051962: positive regulation of nervous system development

R-HSA-6794361: Neurexins and neuroligins

GO:0050808: synapse organization

$\mathrm{GO}: 0031644$ : regulation of neurological system process

GO:0060627: regulation of vesicle-mediated transport

GO:0046717: acid secretion

GO:0008306: associative learning

GO:0021648: vestibulocochlear nerve morphogenesis

GO:0099550: trans-synaptic signaling, modulating synaptic transmission

GO:0016358: dendrite development

GO:0043524: negative regulation of neuron apoptotic process

GO:0051491: positive regulation of filopodium assembly

GO:0050768: negative regulation of neurogenesis

M13: PID ERBB4 PATHWAY

GO:0007613: memory

GO:0050795: regulation of behavior

0003355: multicellular organismal response to stress

GO:0007631: feeding behavior

C

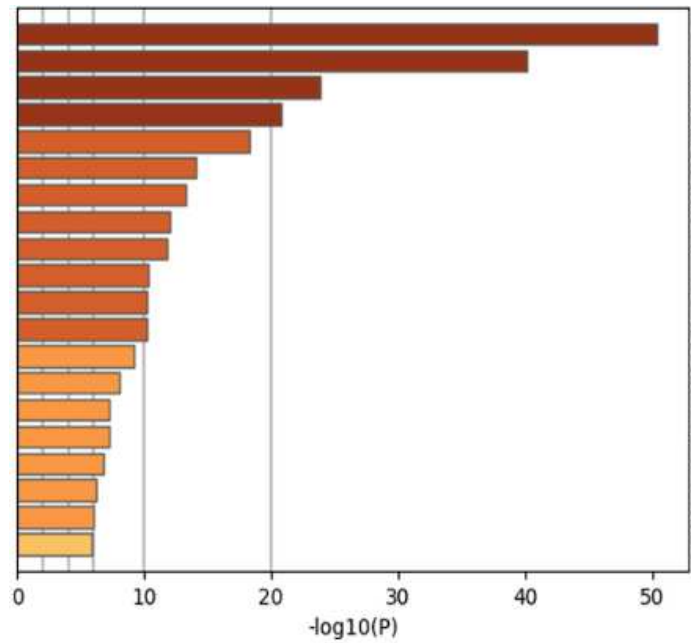

GO:0099537: trans-synaptic signaling

GO:0006836: neurotransmitter transport

GO:0051962: positive regulation of nervous system development

GO:0050808: synapse organization

GO:0007610: behavior

GO:0001504: neurotransmitter uptake

GO:0006865: amino acid transport

GO:0036465: synaptic vesicle recycling

GO:0006906: vesicle fusion

GO:0001764: neuron migration

GO:0048168: regulation of neuronal synaptic plasticity

GO:0060079: excitatory postsynaptic potential

GO:0007420: brain development

GO:0035418: protein localization to synapse

GO:0097091: synaptic vesicle clustering

GO:0016358: dendrite development

GO:0043523: regulation of neuron apoptotic process

GO:0007422: peripheral nervous system development

GO:0007626: locomotory behavior

GO:0015812: gamma-aminobutyric acid transport

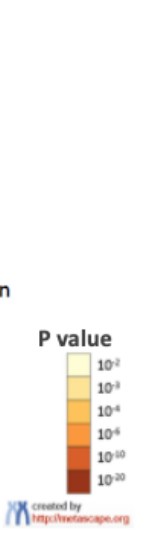

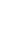

Suppl. Fig. 4: Representation of the biological phenomena in which the DE miRNA in SPERM are involved. The colors of the bars indicate the values of $P$; the darker the colors, the lower the $\mathrm{P}$ value. Analysis was performed using the human database. A: Part 1; B: Part 2; C: Part 3. 


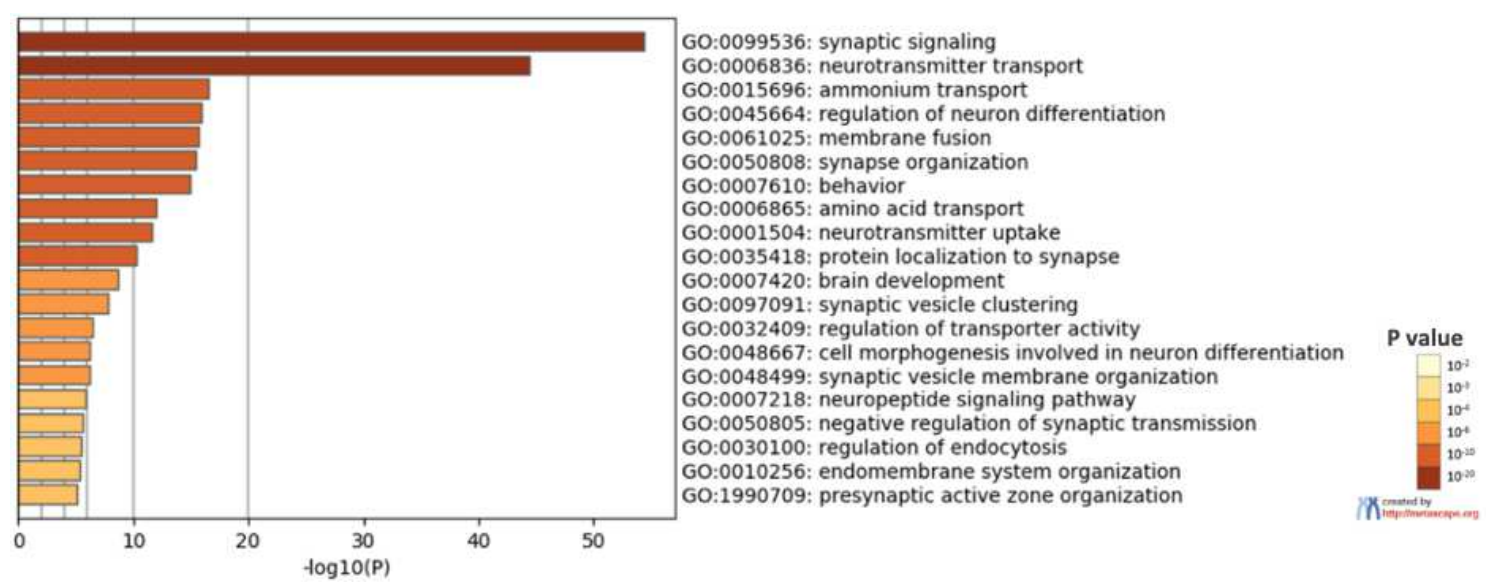

Suppl. Fig. 5: Representation of the biological phenomena in which the DE miRNA in PFC are involved. The colors of the bars indicate the values of $\mathrm{P}$; the darker the colors, the lower the $P$ value. Analysis was performed using the human database.

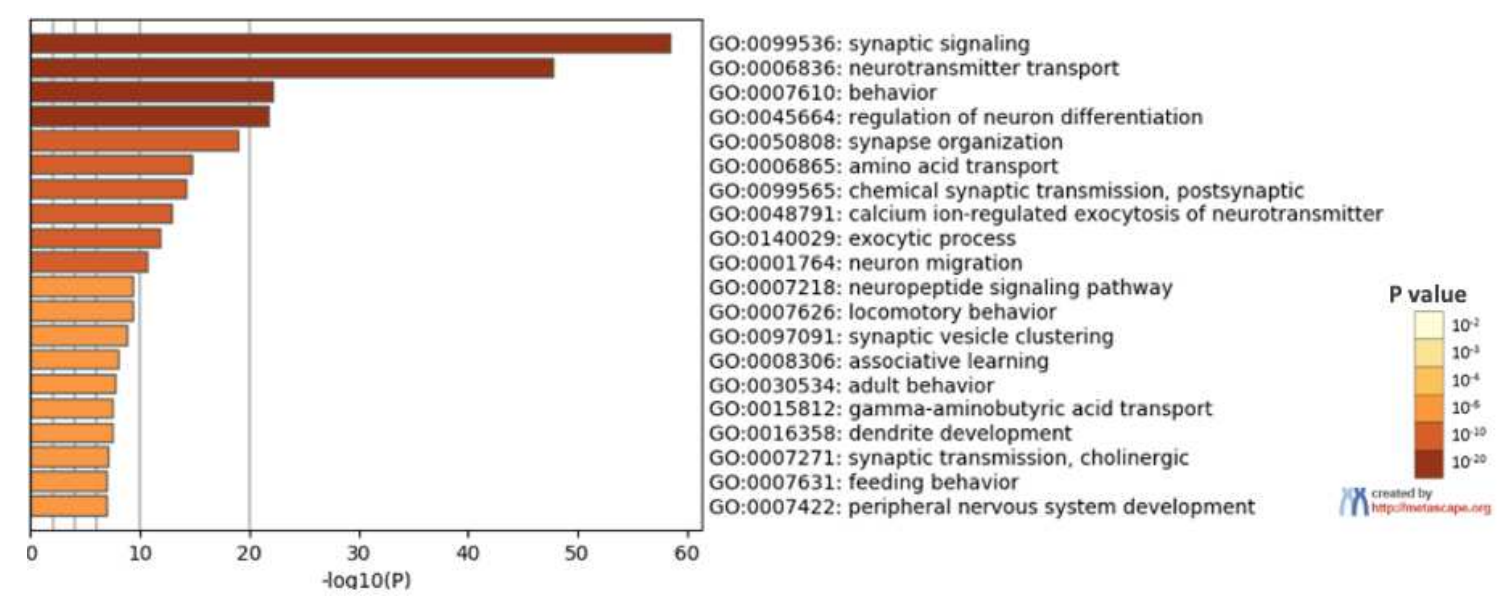

Suppl. Fig. 6: Representation of the biological phenomena in which the DE miRNA in HIPPO are involved. The colors of the bars indicate the values of $P$; the darker the colors, the lower the $\mathrm{P}$ value. Analysis was performed using the human database. 

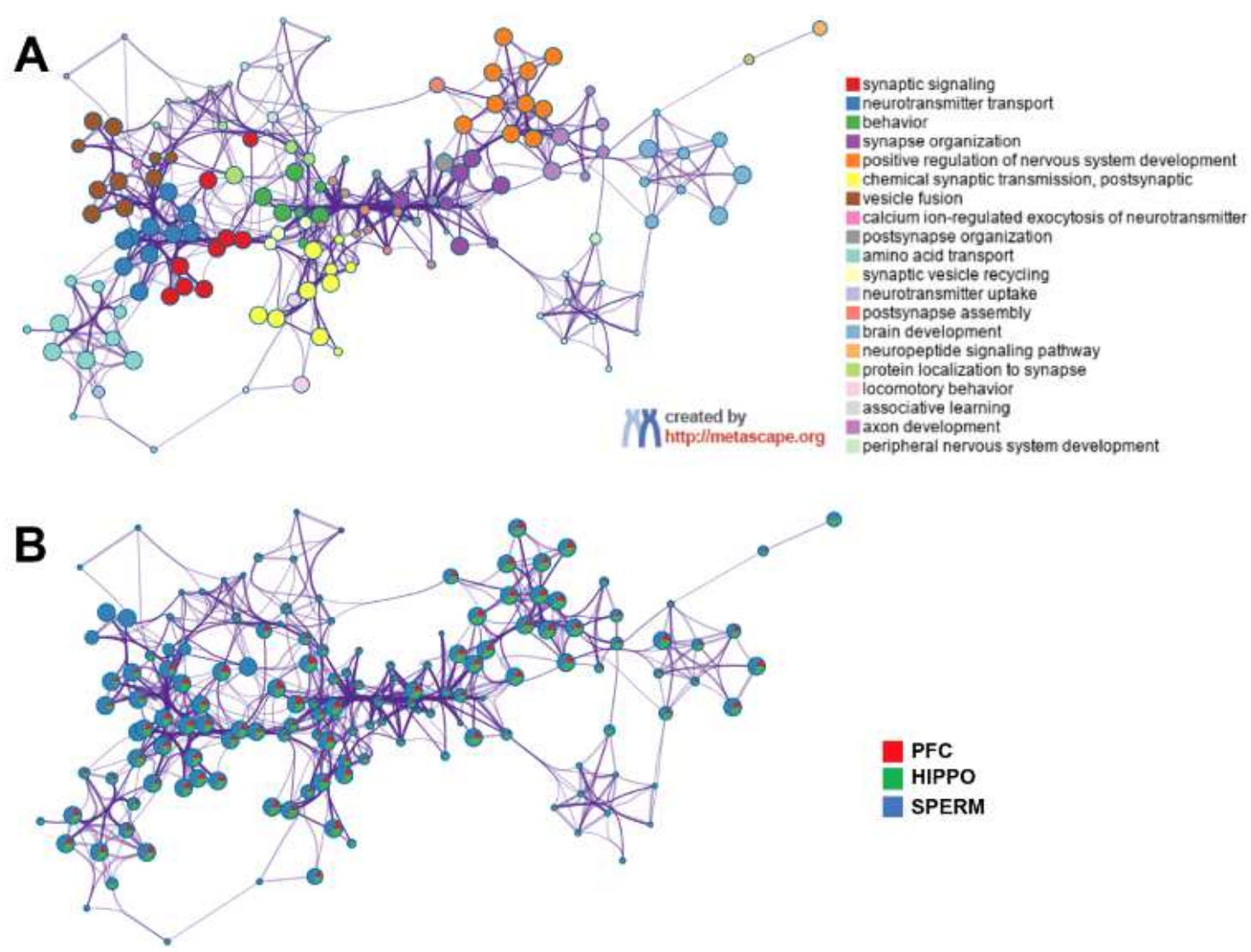

Suppl. Fig. 7: Enrichment network (Gene Ontology Enrichment) in which the nodes represent the hits involved in each biological process. In A, each process is represented by a color. The size of the nodes indicates the amounts of genes involved in the hits and the purple lines between the nodes represent their interactions. Analysis was performed using the human database. In $\mathbf{B}$ the nodes are represented as pie charts. Each portion of the pie represents a type of sample, and the size of each portion represents the proportion of each hit in each type of sample. Each hit includes the genes involved in biological processes, which are identified by the colors in $\mathbf{A}$. 


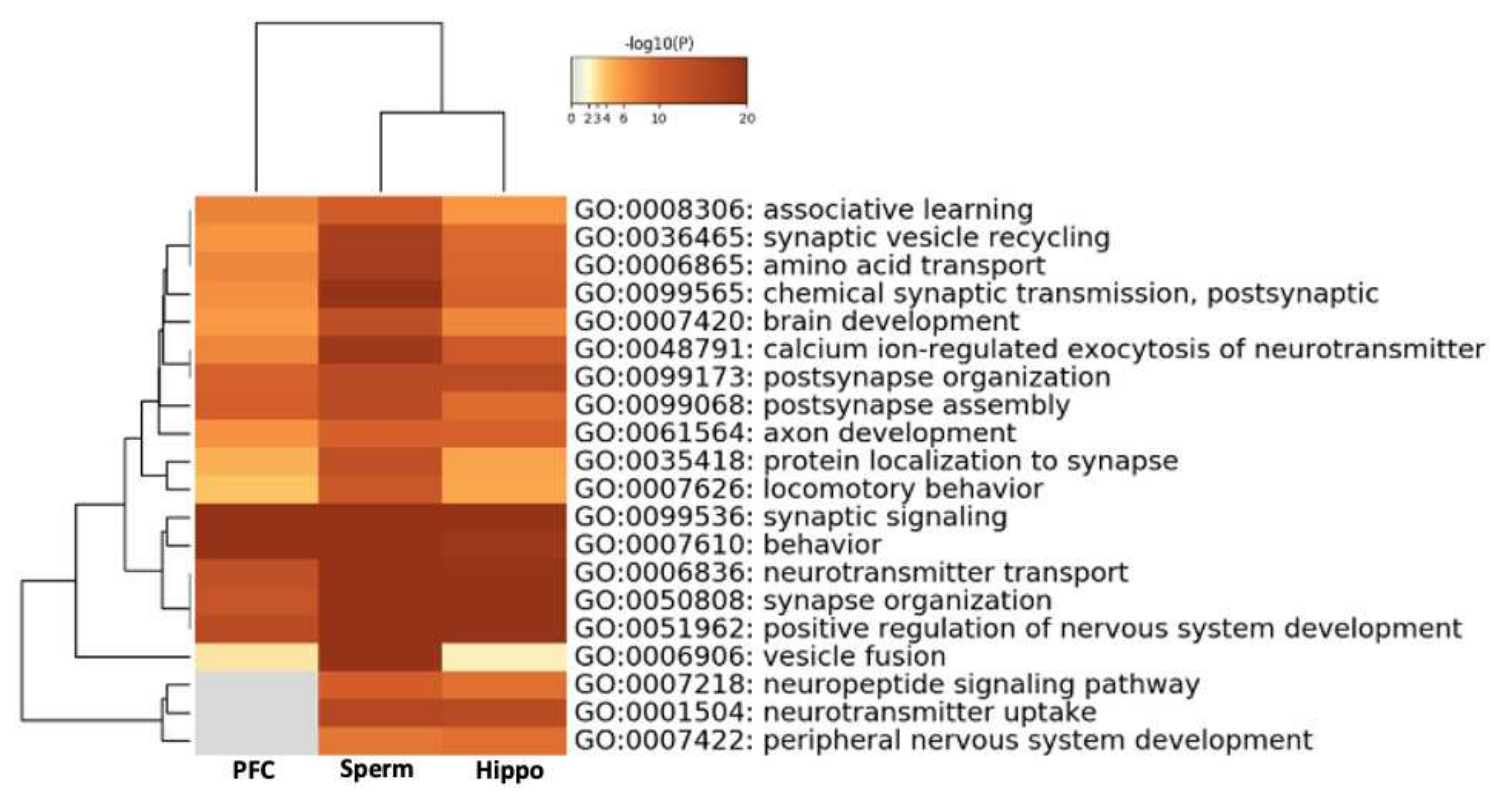

Suppl. Fig. 8: Hierarchical heatmap illustrating the relationship between the identified biological processes and between three type of samples. The colors of the rectangles indicate the $\mathrm{P}$ values; the darker the colors, the lower the value of $\mathrm{P}$. It is observed that there is considerable similarity between the hippocampus and sperm. Behavior, synaptic organization and synaptic signaling appear as processes that could be significantly affected in the three types of sample. Analysis was performed using the human database. 


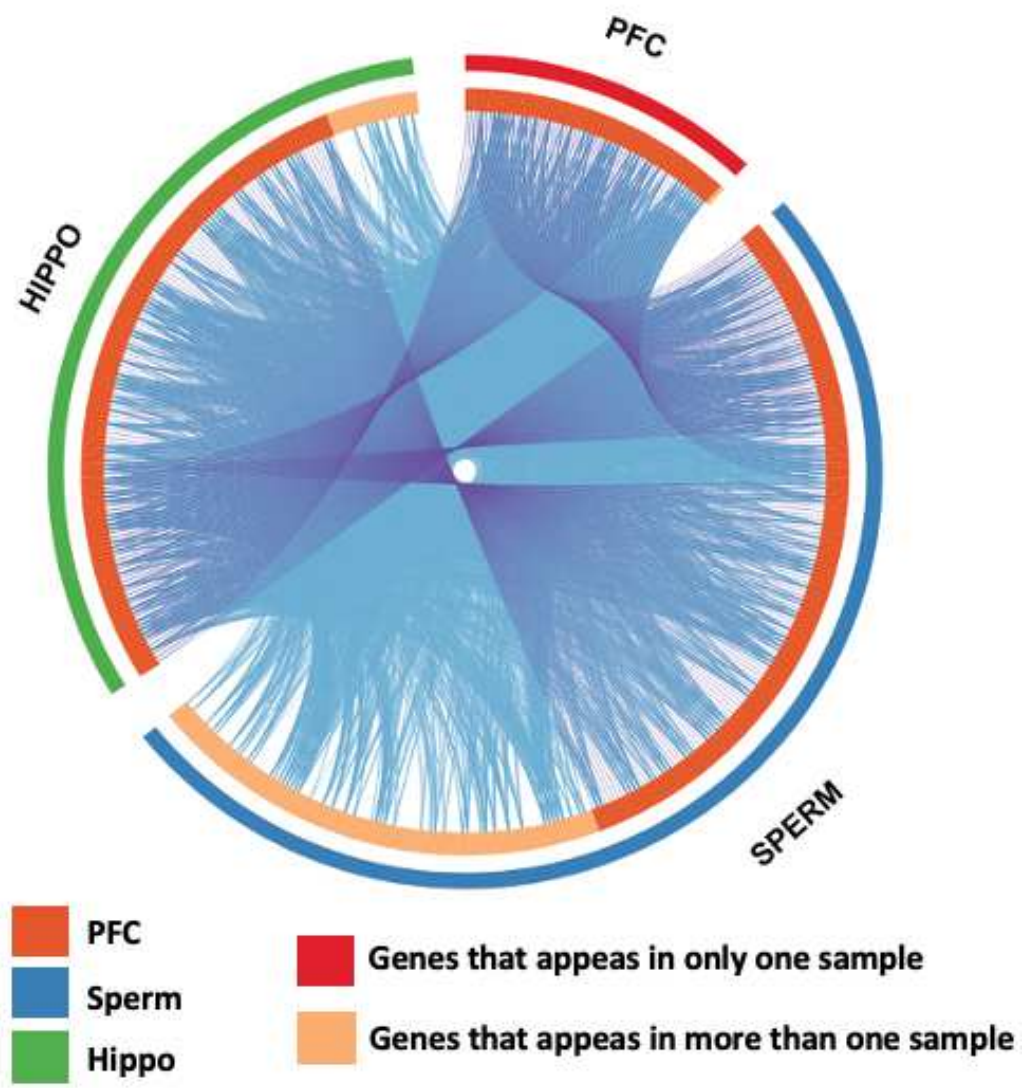

Suppl. Fig. 9: Circus Plot illustrates how differently expressed miRNA target genes coincide between the three types of sample. The outermost arches, represented in blue, red and green, indicate sperm, prefrontal cortex and hippocampus, respectively. The internal arcs, represented in dark orange and light orange, represent the genes that appear in more than one type of sample or in a single type of sample, respectively. The purple lines indicate genes that appear in more than one type of sample. The blue lines link genes that are in the same "gene ontology term" in different types of sample. Analysis was performed using human database.

\section{DISCUSSION}

The use of experimental models to study SCZ is a challenge, since it is an exclusively human psychiatric disorder with complex symptoms. On the other hand, models are important to investigate SCZ aspects that cannot be addressed in clinical studies. In this study we investigated miRNA profile in the prefrontal cortex and hippocampus of SHR with the aim to contribute to search for SCZ models and to the elucidation of epigenetic mechanisms possibly involved in the pathophysiology of this disorder. We also looked at the miRNA profile in the sperm with the aim to provide preliminary data for future studies on epigenetic inheritance of SCZ. 
In the conditions of the present study, five miRNAs showed altered expression in the prefrontal cortex, 13 in the hippocampus and 48 in sperm. Bioinformatic analysis showed the DE miRNA in the SHR are involved in multiple interaction networks with common target genes. The top 20 enriched pathways in which these miRNAs are involved include neurodevelopment, behaviour, memory, learning, and synapsis physiology, all of them known to be affected in SCZ. A recent study also used a rat model of SCZ (methylazoxymethanol acetate model - MAM) to study the expression of possible miRNAs related to schizophrenia ${ }^{25}$ and observed changes in the expression of genes related to synaptic chemical transmission, cognition, and inflammatory response in the MAM rats in relation to controls. As observed for MAM rats in the study by Du and collaborators ${ }^{25}$, here we also indicate that SHR can be an interesting model for SCZ since they might be able to reproduce impairment of hippocampus and prefrontal cortex, which is related to SCZ. Additionally, this model contributes to the comprehension of epigenetic aspects of this disorder.

Among the molecular aspects of schizophrenia, genetic aspects have been the focus of most studies. More recently, the epigenetic approach has been also gaining attention. Differential methylation pattern of genes identified as relevant for SCZ, such as DISC1 and BDNF, for example, have already been described in SCZ patients ${ }^{26-29}$. Broader studies have also reported alteration in bulk DNA methylation in patients diagnosed with SCZ $28,30,31$. As previously mentioned, dysregulation of miRNA expression has also been demonstrated in SCZ. Differential expression of miR-137 has been reported in different studies ${ }^{16,32,33,34}$ and this miRNA has been suggested as a potential biomarker for this disorder. Other miRNAs have been reported as differentially expressed in SCZ patients ${ }^{16,35-39}$. However, a considerable discrepancy in the results is observed among the studies. This variability might result from several factors such as the type of sample (blood, different regions of the brain) and the type of techniques applied, what indicates that a wide miRNA analysis providing the profile of altered miRNAs is important. 
The function of miRNAs is mostly related to their interaction with target mRNAs, which represent a target gene. Thus, the association of a specific miRNA to a process or disease is given by the role of the target gene in that process or disease. This can be addressed using microRNA Target Prediction databases such as TargetScan and miRDB, followed by gene ontology and gene enrichment analyses. In the "Translational Analysis" of the Results section of this manuscript we mentioned that we found 12 genes related to schizophrenia that were targeted by the miRNA differentially expressed in the prefrontal cortex and in the hippocampus. Therefore, we were able to suggest that the miRNA differentially expressed in these regions can be related to schizophrenia. We found that miR-130a, miR-664, miR-31, miR-150, miR-30a, miR-17, miR-26b, miR-499, miR-15b and miR-16 have been reported as relevant for this disorder, as reviewed by Beveridge and Cairns ${ }^{1}$. We did not find a direct association of miR-551b, miR-465, miR-451, miR-667, miR-3546 and miR-344b, miR-3589 and miR3562 with schizophrenia. However, all these miRNAs are related to neurodevelopmental and/or neurophysiological processes, as shown by our target analysis (miRDB and TargetScan) or by the literature ${ }^{40-43}$.

Among the DE miRNAs, miR-130a showed differential expression in the PFC and in the sperm. This miRNA is involved in important processes during prenatal neurogenesis, such as neurite growth and dendritic spine density ${ }^{44}$ and has also been associated with SCZ ${ }^{45}$. The expression of miR-130a has already been reported in sperm of different species, but its gametic and the early embryonic function has not yet been well defined. It is known that during the beginning of embryonic development, miR-130a acts as a regulator in physiological processes related to mesodermal specification, angiogenesis ${ }^{46}$ and maternal-embryonic transition ${ }^{47}$, which is a crucial moment when the embryo starts to control its own development through the activation of its genome. The importance of miRNAs from sperm for the early development of the embryo has already been indicated ${ }^{48,49}$. Interestingly, the expression of the same miRNA can be present in different stages of embryonic development, assuming 
different regulatory functions according to each of these stages. Thus, it would be interesting to investigate whether altering the expression of miR-130a and of the other DE miRNAs found in sperm can affect t embryonic and nervous system development. Studies about paternal epigenetic inheritance of psychiatric disorders are relatively recent and it is not known whether a correspondence between the sperm and the brain is determinant to the transmission of the altered behaviour, since the miRNAs altered in the sperm will be transferred to the embryo and will be soon be degraded. Different studies have shown that changes in miRNA expression in mouse sperm caused by trauma and stress can lead to behaviour alterations in their offspring ${ }^{50-53}$. However, no data were found in the literature regarding the possible influence of sperm miRNAs on schizophrenia. The fact that the same SCZ-related miRNA (miR-130a) was altered in the sperm and in the brain reinforces the importance of studying paternal miRNA inheritance in SCZ. Our future aim is to investigate the possible influence of the altered sperm miRNA on the development of the nervous system and in the manifestation of the SCZ-like behaviour in the SHR.

As previously mentioned, one of the great challenges to establish experimental models for psychiatric disorders is the fact that these disorders are human manifestations and their diagnosis is based on consciousness parameters. On the other hand, many biological characteristics are conserved across mammalian species, making possible to carry out translational analyses. Consequently, it is important to obtain information about the biological aspects of proposed models. In our study, the enriched pathways generated by Metascape analysis using human and rat databases were related to neurological processes such as synapse physiology, behaviour, brain development, learning, and memory. These data show that the targets of the DE miRNAs are similar in rats and humans, as are the processes in which they are involved. And more importantly, target genes for these DE miRNAs have been described as altered in SCZ patients, as indicated by the analysis using the SZDB 
database. These data reinforce results from previous studies suggesting the SHR as a good model for SCZ ${ }^{54}$.

In conclusion, the data presented here provide information regarding miRNAs profile in prefrontal cortex, hippocampus, and sperm of SHR aiming to contribute to the characterization of these rats as a model for molecular and epigenetic studies on SCZ. Considering SCZ presents an important environmental component ${ }^{55}$, which is modulated by epigenetic alterations, including miRNA expression, these data can serve as basis for future studies using SHR in this field. Since $70 \%$ of the miRNAs described in mammals are present in the brain ${ }^{56}$, information about miRNA profile related to this disorder can be an important contribution to the comprehension of this disorder and to provide new perspectives on diagnostic strategies and treatment. Finally, our data direct us to future studies about the consequences of SCZ-related miRNA dysregulation in the sperm to the offspring.

\section{METHODS}

\section{Animals and Sample Collection}

Male Wistar rats (Rattus norvegicus albinus) and Spontaneously Hypertensive Rats (SHR) obtained from the Centre for Development of Animal Models for Medicine and Biology (CEDEME) were used for this study. Adult animals (90-100 days old) were kept in plastic cages under a $12-12 \mathrm{~h}$ light/dark cycle at $23-25{ }^{\circ} \mathrm{C}$. Food and water were allowed ad libitum. The animals were separated into two groups: control (CT), composed by Wistar rats $(n=9)$; and SHR, composed by SHR $(n=9)$. Euthanasia was performed by anaesthesia/analgesia (xylazine/ketamine, $10 \mathrm{mg} / \mathrm{Kg}$ and $100 \mathrm{mg} / \mathrm{Kg}$, respectively) followed by cardiac incision. Immediately after euthanasia, the sperm (SPERM), the prefrontal cortex (PFC) and the hippocampus (HIPPO) were collected and submitted to microRNA isolation, as described later. 
All animal handling was carried out according to the National Institutes of Health guide for the care and use of Laboratory animals. This study was approved by the Committee for Animal Use (CEUA/UNIFESP - $n^{\circ}$ 6315260117) of the Universidade Federal de São Paulo (UNIFESP).

\section{miRNA extraction, library construction and sequencing}

The extraction of miRNA from sperm, hippocampus and prefrontal cortex was performed using the mirVana miRNA Isolation kit (Cat. AM1561, Thermo Fisher Scientific Carlsbad, California, USA) according to the manufacturer's instructions. miRNA quantification was performed in Bioanalyzer 2100 (Agilent Technologies) using the Small RNA Kit (Cat. 5067-1548, Agilent - Santa Clara, California, USA). Libraries were constructed using the Ion Total RNA-seq kit v2 for Small RNA Libraries (Cat. 4475936, Thermo Fisher Scientific - Carlsbad, California, USA). Barcoded libraries were quantified in the Bioanalyzer 2100 (Agilent Technologies) using the High Sensitivity DNA Chip (Cat 5067-4626, Agilent Technologies - Santa Clara, California, USA). Clonal amplification of the libraries was carried out using lon $540^{\mathrm{TM}}$ kit Chef (Cat. A30011, Thermo Fisher Scientific - Carlsbad, California, USA) and sequenced using the Ion 540 тм Chip (Cat. A27766, Thermo Fisher Scientific - Carlsbad, California, USA) in the lon S5 Semiconductor Sequencer according to the instructions provided by the manufacturer.

\section{Differential Expression Analysis}

The analysis of miRNA expression was performed using Partek® Flow ${ }^{\circledR}$ software following the pipeline indicated for miRNA. Torrent Mapping Alignment Program (TMAP) was used for sequence mapping, then it was performed miRNA quantification using annotation model (Partek E/M) followed by a normalization step using counts per million (CPM) method followed by the addition of 1.0E-11. GSA differential analysis algorithm was applied in order to determine differentially expressed 
(DE) miRNA amongst studied groups. miRNA presenting fold change (FC) $<-2$ or $>2$ and $p<0,05$ were selected for further analysis.

\section{Target genes and pathway analyses}

The DE miRNAs target genes were predicted using TargetScan (version 7.2) and miRDB ${ }^{57,58}$ rat databases and only the ones that appeared in both platforms were considered. Gene-miRNA Interaction networks were obtained through Cytoscape ${ }^{\circledR}$ (version 3.8.0). Metascape ${ }^{59}$ enrichment analysis was used to predict the pathways in which the DE miRNA are involved.

\section{Translational analyses}

To help to validate the information obtained from the SHR model, the DE miRNA were also analysed considering the human databases with the aim to investigate whether the same miRNA plays a conserved role in rats and humans. For this, TargetScan (version 7.2) and miRDB were also used to obtain the predicted target genes, followed by Cytoscape ${ }^{\circledR}$ and Metascape softwares analyses. The relationship of the target genes with schizophrenia was investigated using the Schizophrenia Database (SZDB) version $2.0^{60}$.

\section{REFERENCES}

1 Beveridge N.J., Cairns M.J.. MicroRNA dysregulation in schizophrenia. Neurobiol Dis.;46(2):263-271. doi:10.1016/j.nbd.2011.12.029 (2012).

2 Wang J., Wang Y., Yang J., Huang Y.. microRNAs as novel biomarkers of schizophrenia (Review). Exp Ther Med.;8(6):1671-1676. doi:10.3892/etm.2014.2014 (2014). 
3 Mellios N., Huang H.S., Baker S.P., Galdzicka M., Ginns E., Akbarian S.. Molecular determinants of dysregulated GABAergic gene expression in the prefrontal cortex of subjects with schizophrenia. Biol Psychiatry.;65(12):1006-1014. doi:10.1016/j.biopsych.2008.11.019 (2009).

4 Perkins D.O. et al. microRNA expression in the prefrontal cortex of individuals with schizophrenia and schizoaffective disorder. Genome Biol.;8(2):R27. doi:10.1186/gb2007-8-2-r27 (2007).

5 Santarelli D.M., Beveridge N.J., Tooney P.A., Cairns M.J.. Upregulation of dicer and microRNA expression in the dorsolateral prefrontal cortex Brodmann area 46 in schizophrenia. Biol Psychiatry.;69(2):180-187. doi:10.1016/j.biopsych.2010.09.030 (2011).

$6 \mathrm{Kim} \mathrm{A.H.} \mathrm{et} \mathrm{al.} \mathrm{MicroRNA} \mathrm{expression} \mathrm{profiling} \mathrm{in} \mathrm{the} \mathrm{prefrontal} \mathrm{cortex} \mathrm{of} \mathrm{individuals}$ affected with schizophrenia and bipolar disorders. Schizophr Res.;124(1-3):183-191. doi:10.1016/j.schres.2010.07.002 (2010).

7 Fineberg S.K., Kosik K.S., Davidson B.L.. MicroRNAs potentiate neural development. Neuron.;64(3):303-309. doi:10.1016/j.neuron.2009.10.020 (2009).

8 Atanur S.S. et al. The genome sequence of the spontaneously hypertensive rat: Analysis and functional significance. Genome Res.;20(6):791-803. doi:10.1101/gr.103499.109 (2010). 
9 Zhang-James Y.., Middleton F.A., Faraone S.V.. Genetic architecture of Wistar-Kyoto rat and spontaneously hypertensive rat substrains from different sources. Physiol Genomics.;45(13):528-538. doi:10.1152/physiolgenomics.00002.2013 (2013).

10 Meneses A., Perez-Garcia G., Ponce-Lopez T., Tellez R., Gallegos-Cari A., Castillo C.. Spontaneously hypertensive rat (SHR) as an animal model for ADHD: a short overview. Rev Neurosci.;22(3):365-371. doi:10.1515/RNS.2011.024. (2011).

11 Russell V.A. Neurobiology of animal models of attention-deficit hyperactivity disorder. J Neurosci Methods.;161(2):185-198. doi:10.1016/j.jneumeth.2006.12.005. (2007).

12 Sagvolden T., Metzger M.A., Schiørbeck H.K., Rugland A.L., Spinnangr I., Sagvolden G.. The spontaneously hypertensive rat (SHR) as an animal model of childhood hyperactivity (ADHD): changed reactivity to reinforcers and to psychomotor stimulants. Behav Neural Biol. ;58(2):103-112. doi:10.1016/0163-1047(92)90315-u (1992)

13 Niigaki S.T. et al. Young spontaneously hypertensive rats (SHRs) display prodromal schizophrenia-like behavioral abnormalities. Prog Neuropsychopharmacol Biol Psychiatry:;90:169-176. doi:10.1016/j.pnpbp.2018.11.020 (2019).

14 Peres F.F. et al. Cannabidiol Administered During Peri-Adolescence Prevents Behavioral Abnormalities in an Animal Model of Schizophrenia. Front Pharmacol. 2018;9:901. Published 2018 Aug 21. doi:10.3389/fphar.2018.00901 (2018). 
15 Sagvolden T., Sergeant J.A.. Attention deficit/hyperactivity disorder--from brain dysfunctions to behaviour. Behav Brain Res.;94(1):1-10 (1998).

16 Levin R. et al. Spontaneously Hypertensive Rats (SHR) present deficits in prepulse inhibition of startle specifically reverted by clozapine. Prog Neuropsychopharmacol Biol Psychiatry.;35(7):1748-1752. doi:10.1016/j.pnpbp.2011.06.003 (2011).

17 Diana M.C. et al. Sodium nitroprusside is effective in preventing and/or reversing the development of schizophrenia-related behaviors in an animal model: The SHR strain. CNS Neurosci Ther.;24(7):624-632. doi:10.1111/cns.12852 (2018).

18 Cao T., Zhen X.C.. Dysregulation of miRNA and its potential therapeutic application in schizophrenia. CNS Neurosci Ther. 2018;24(7):586-597. doi:10.1111/cns.12840 (2018).

$19 \mathrm{Hu}$ Z. et al. Temporal dynamics of miRNAs in human DLPFC and its association with miRNA dysregulation in schizophrenia [published correction appears in Transl Psychiatry. 2019 Sep 23;9(1):237]. Trans/ Psychiatry. 2019;9(1):196. Published 2019 Aug 20. doi:10.1038/s41398-019-0538-y (2019).

$20 \mathrm{Ma} \mathrm{J}$. et al. Identification of miR-22-3p, miR-92a-3p, and miR-137 in peripheral blood as biomarker for schizophrenia. Psychiatry Res. 2018;265:70-76. doi:10.1016/j.psychres.2018.03.080 (2018).

21 Edwards M.J.. Hyperthermia in utero due to maternal influenza is an environmental risk factor for schizophrenia. Congenit Anom (Kyoto). 2007;47(3):84-89. doi:10.1111/j.1741-4520.2007.00151.x (2007). 
22 Markham J.A., Koenig J.I.. Prenatal stress: role in psychotic and depressive diseases. Psychopharmacology (Berl).;214(1):89-106. doi:10.1007/s00213-010-2035-0 (2011).

23 Cruz-Rizzolo R.J. et al. Protein malnutrition during gestation and early life decreases neuronal size in the medial prefrontal cortex of post-pubertal rats. IBRO Rep. 2017;3:65-71. Published 2017 Oct 6. doi:10.1016/j.ibror.2017.08.002 (2017).

24 Pugliese V. et al. Maternal stress, prenatal medical illnesses and obstetric complications: Risk factors for schizophrenia spectrum disorder, bipolar disorder and major depressive disorder. Psychiatry Res. 2019;271:23-30. doi:10.1016/j.psychres.2018.11.023 (2019).

25 Du Y., Li X.S., Chen L., Chen G.Y., Cheng Y.. A Network Analysis of Epigenetic and Transcriptional Regulation in a Neurodevelopmental Rat Model of Schizophrenia With Implications for Translational Research. Schizophr Bull.;46(3):612-622. doi:10.1093/schbul/sbz114 (2020).

26 Ikegame T. et al. DNA methylation analysis of BDNF gene promoters in peripheral blood cells of schizophrenia patients. Neurosci Res.;77(4):208-214. doi:10.1016/j.neures.2013.08.004 (2013).

27 Teroganova N., Girshkin L., Suter C.M., Green M.J.. DNA methylation in peripheral tissue of schizophrenia and bipolar disorder: a systematic review. BMC Genet. 2016;17:27. Published 2016 Jan 25. doi:10.1186/s12863-016-0332-2 (2016). 
28 Hannon E. et al. An integrated genetic-epigenetic analysis of schizophrenia: evidence for co-localization of genetic associations and differential DNA methylation. Genome Biol:;17(1):176. Published 2016 Aug 30. doi:10.1186/s13059016-1041-x (2016).

29 McCartney D.L. et al. Altered DNA methylation associated with a translocation linked to major mental illness. NPJ Schizophr.;4(1):5. Published 2018 Mar 19. doi:10.1038/s41537-018-0047-7 (2018).

30 Alelú-Paz R. et al. Epigenetics in Schizophrenia: A Pilot Study of Global DNA Methylation in Different Brain Regions Associated with Higher Cognitive Functions. Front Psychol. 2016;7:1496. Published 2016 Sep 30. doi:10.3389/fpsyg.2016.01496 (2016).

$31 \mathrm{Li} \mathrm{Y}$. et al. Genome-wide methylome analyses reveal novel epigenetic regulation patterns in schizophrenia and bipolar disorder. Biomed Res Int:;2015:201587. doi:10.1155/2015/201587 (2015).

32 Arakawa $\mathrm{Y}$. et al. Transgenic mice overexpressing miR-137 in the brain show schizophrenia-associated behavioral deficits and transcriptome profiles. PLoS One. 2019;14(7):e0220389. Published 2019 Jul 30. doi:10.1371/journal.pone.0220389 (2019).

33 Ma G. et al. Association of a miRNA-137 polymorphism with schizophrenia in a Southern Chinese Han population. Biomed Res Int.;2014:751267. doi:10.1155/2014/751267 (2014). 
$34 \mathrm{Wu}$ S. et al. MicroRNA-137 Inhibits EFNB2 Expression Affected by a Genetic Variant and Is Expressed Aberrantly in Peripheral Blood of Schizophrenia Patients. EBioMedicine.;12:133-142. doi:10.1016/j.ebiom.2016.09.012 (2016).

$35 \mathrm{Yu}$ H.C. et al. Alterations of miR-132 are novel diagnostic biomarkers in peripheral blood of schizophrenia patients. Prog Neuropsychopharmacol Biol Psychiatry. 2015;63:23-29. doi:10.1016/j.pnpbp.2015.05.007 (2015).

36 Grossi I, Salvi A, Abeni E, Marchina E, De Petro G. Biological Function of MicroRNA193a-3p in Health and Disease. Int J Genomics. 2017;2017:5913195. doi:10.1155/2017/5913195.

37 Mor E., Kano S., Colantuoni C., Sawa A., Navon R., Shomron N.. MicroRNA-382 expression is elevated in the olfactory neuroepithelium of schizophrenia patients. Neurobiol Dis.;55:1-10. doi:10.1016/j.nbd.2013.03.011 (2013).

38 Cattane N. et al. Identification of a miRNAs signature associated with exposure to stress early in life and enhanced vulnerability for schizophrenia: New insights for the key role of miR-125b-1-3p in neurodevelopmental processes. Schizophr Res. ;205:6375. doi:10.1016/j.schres.2018.07.030 (2019)

39 Wei $\mathrm{H}$. et al. Detection of circulating miRNA levels in schizophrenia. Am J Psychiatry. ;172(11):1141-1147. doi:10.1176/appi.ajp.2015.14030273 (2015).

40 Jovičić A. et al. Comprehensive expression analyses of neural cell-type-specific miRNAs identify new determinants of the specification and maintenance of neuronal phenotypes. J Neurosci. ;33(12):5127-5137. doi:10.1523/JNEUROSCI.0600-12.2013 (2013). 
41 Trattnig C. et al. MicroRNA-451a overexpression induces accelerated neuronal differentiation of Ntera2/D1 cells and ablation affects neurogenesis in microRNA-451aI- mice. PLoS One. 2018;13(11):e0207575. Published 2018 Nov 21. doi:10.1371/journal.pone.0207575 (2018).

42 Boese A.S. et al. MicroRNA abundance is altered in synaptoneurosomes during prion disease. Mol Cell Neurosci. ;71:13-24. doi:10.1016/j.mcn.2015.12.001 (2016).

43 Leong J.W., Abdullah S., Ling K.H., Cheah P.S.. Spatiotemporal Expression and Molecular Characterization of miR-344b and miR-344c in the Developing Mouse Brain. Neural Plast. ;2016:1951250. doi:10.1155/2016/1951250 (2016).

44 Zhang Y. et al. MiR-130a regulates neurite outgrowth and dendritic spine density by targeting MeCP2. Protein Cell. ;7(7):489-500. doi:10.1007/s13238-016-0272-7 (2016).

45 Beveridge N.J., Gardiner E., Carroll A.P., Tooney P.A., Cairns M.J.. Schizophrenia is associated with an increase in cortical microRNA biogenesis. Mol Psychiatry.;15(12):1176-1189. doi:10.1038/mp.2009.84 (2010).

46 Singh B.N., et al. The Etv2-miR-130a Network Regulates Mesodermal Specification. Cell Rep. ;13(5):915-923. doi:10.1016/j.celrep.2015.09.060 (2015).

47 Mondou E., Dufort I., Gohin M., Fournier E., Sirard M.A.. Analysis of microRNAs and their precursors in bovine early embryonic development. Mol Hum Reprod. ;18(9):425-434. doi:10.1093/molehr/gas015 (2012). 
48 Hua M. et al. Identification of small non-coding RNAs as sperm quality biomarkers for in vitro fertilization. Cell Discov. 2019;5:20. Published 2019 Apr 9. doi:10.1038/s41421-019-0087-9 (2019).

49 Yuan S. et al. Sperm-borne miRNAs and endo-siRNAs are important for fertilization and preimplantation embryonic development. Development. ;143(4):635-647. doi:10.1242/dev.131755 (2016).

50 Chan J.C. et al. Reproductive tract extracellular vesicles are sufficient to transmit intergenerational stress and program neurodevelopment. Nat Commun. ;11(1):1499. Published 2020 Mar 20. doi:10.1038/s41467-020-15305-w (2020).

51 Dickson D.A., et al. Reduced levels of miRNAs 449 and 34 in sperm of mice and men exposed to early life stress. Trans/ Psychiatry. 2018;8(1):101. Published 2018 May 23. doi:10.1038/s41398-018-0146-2 (2018).

52 Gapp K. et al. Implication of sperm RNAs in transgenerational inheritance of the effects of early trauma in mice. Nat Neurosci. ;17(5):667-669. doi:10.1038/nn.3695 (2014).

53 Rodgers A.B., Morgan C.P., Leu N.A., Bale T.L.. Transgenerational epigenetic programming via sperm microRNA recapitulates effects of paternal stress. Proc Natl Acad Sci U S A. ;112(44):13699-13704. doi:10.1073/pnas.1508347112 (2015).

54 Calzavara M.B. et al. Neuroleptic drugs revert the contextual fear conditioning deficit presented by spontaneously hypertensive rats: a potential animal model of emotional context processing in schizophrenia?. Schizophr Bull. 2009;35(4):748-759. doi:10.1093/schbul/sbn006 (2009). 
55 van Dongen J., Boomsma D.I.. The evolutionary paradox and the missing heritability of schizophrenia. Am J Med Genet B Neuropsychiatr Genet. ;162B(2):122136. doi:10.1002/ajmg.b.32135 (2013).

56 Chen W., Qin C.. General hallmarks of microRNAs in brain evolution and development. RNA Biol. ;12(7):701-708. doi:10.1080/15476286.2015.1048954 (2015).

57 Liu W., Wang X. Prediction of functional microRNA targets by integrative modeling of microRNA binding and target expression data. Genome Biol. 2019;20(1):18. Published 2019 Jan 22. doi:10.1186/s13059-019-1629-z (2019).

58 Chen Y., Wang X.. miRDB: an online database for prediction of functional microRNA targets. Nucleic Acids Res. 2020;48(D1):D127-D131. doi:10.1093/nar/gkz757 (2020).

59 Zhou Y. et al. Metascape provides a biologist-oriented resource for the analysis of systems-level datasets. Nat Commun. 2019;10(1):1523. Published 2019 Apr 3. doi:10.1038/s41467-019-09234-6 (2019).

60 Wu Y., Yao Y.G., Luo X.J.. SZDB: A Database for Schizophrenia Genetic Research. Schizophr Bull.;43(2):459-471. doi:10.1093/schbul/sbw102 (2017).

\section{ACKNOWLEDGEMENTS}

The authors thank Ph.D student Camila Albuquerque Pinto and Dr. Leonardo Caires for technical support in miRNA sequencing. 


\section{COMPETING INTERESTS}

Authors declared no competing interests.

\section{FINANTIAL SUPPORT}

This work was supported by Fundação de Amparo à Pesquisa do Estado de São Paulo (FAPESP) Prof. No. 2018/01473-3; Coordenação de Aperfeiçoamento de Pessoal de Nível Superior (CAPES) - Finance code 001. 


\section{Figures}
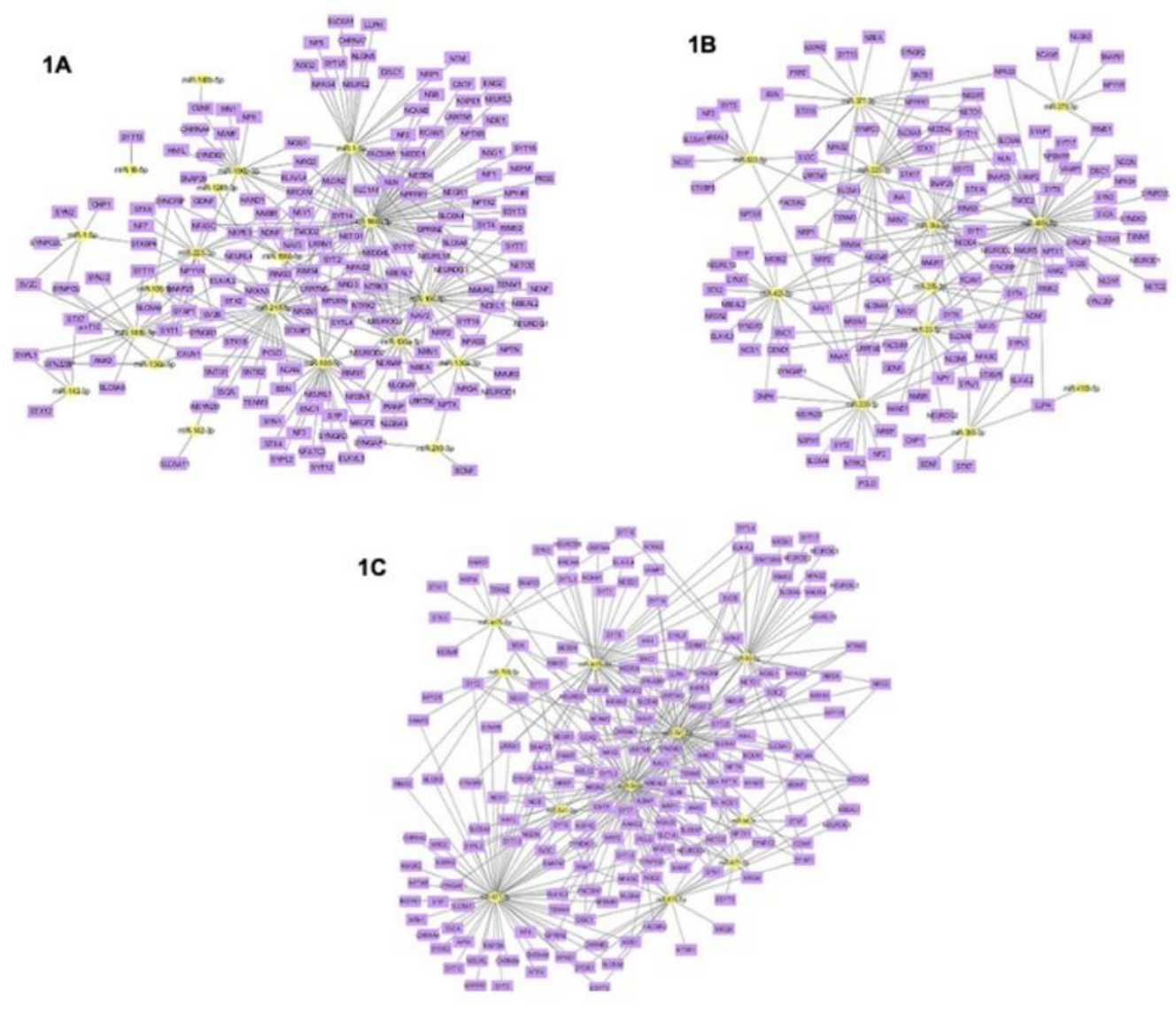

1D
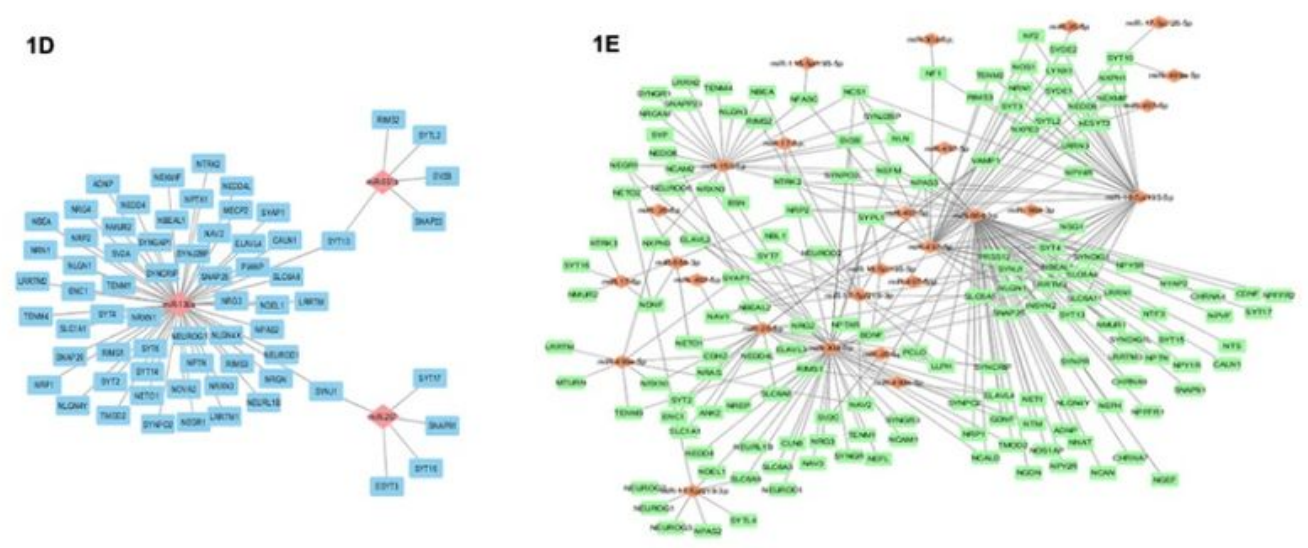

Figure 1

Interaction map of the DE miRNAs (diamonds) and their target genes (rectangles) - in SPERM, part 1 (A), 2 (B) and 3 (C); CPF (D) and HIPPO (E). 


\section{A}

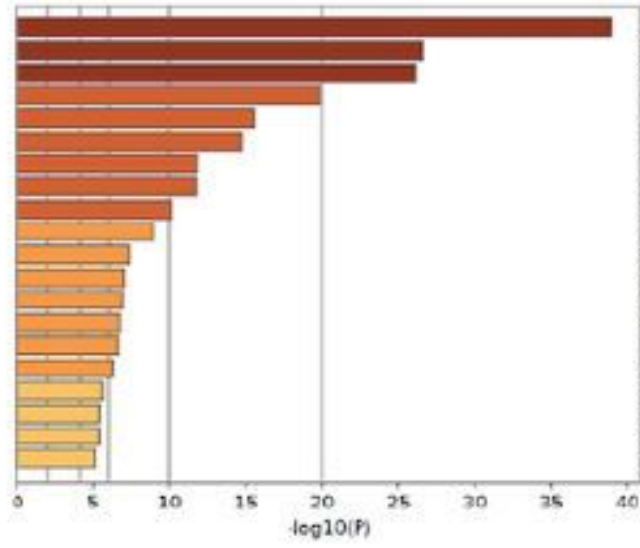

60:0006836: neurotransmitter transport

60:0050804: moduletion of chemical synastic transmissicn 00.0050808: synapse orgarization

G0.0051962: postive reguistion of nervous system devolepment

00:0022406: memberne dodking

G0:0051668; localizetion within membrane

60:0031644: regulation of neurolocical system process

G0.0097091: synaptc vesicle clustering

60:0036465: synaptc wesicle recycing

60:0001764: neuron migration

60:0098917: retrograde trans-syneptic signaling

GO:0046879: hormose secretion

$60-0050772$ : pos tive reguletion of axonogeneg is

GO:C016358: dendrito development

90.0099540; trans-smaptic signaling by neurcpeptide

60:0001504: nourotransmitter uptake

60:0032409: regulaten of transporter activity

00:00A0160; regulaton of reurone aynaptc plosticity

GO. 0090128: regulaton of synapse maturation

2B

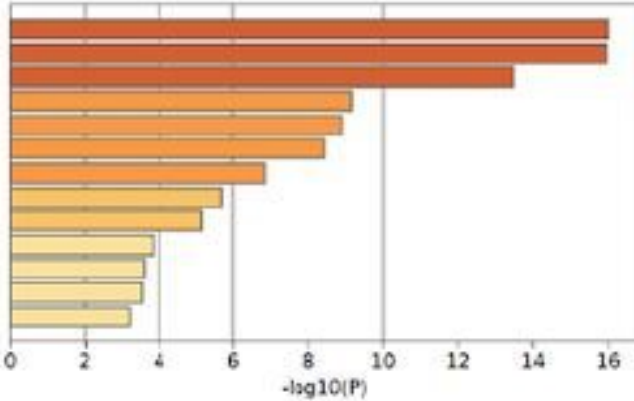

60:0067269: neurotransmizter secretion

00:0051962: positive regulation of nervous systers development

60:0050804: modulation of chemical synaptic transmission

60:0140029: exocytic process

60:0067610: behavict

60:0051668: ocelization within membrane

$60: 00 \% 0100$ : regulation of esdocytesis

60:0016358: dendrite develppment

60:0012783: preganglionic sarasympathetc fiber develepment

$60: 0067626$ : loccmetory behovior

60:0035176: social bohavior

60:00:7613: memory

GO:0050768: negstive regulation of neurogenesis

\section{$2 \mathrm{C}$}

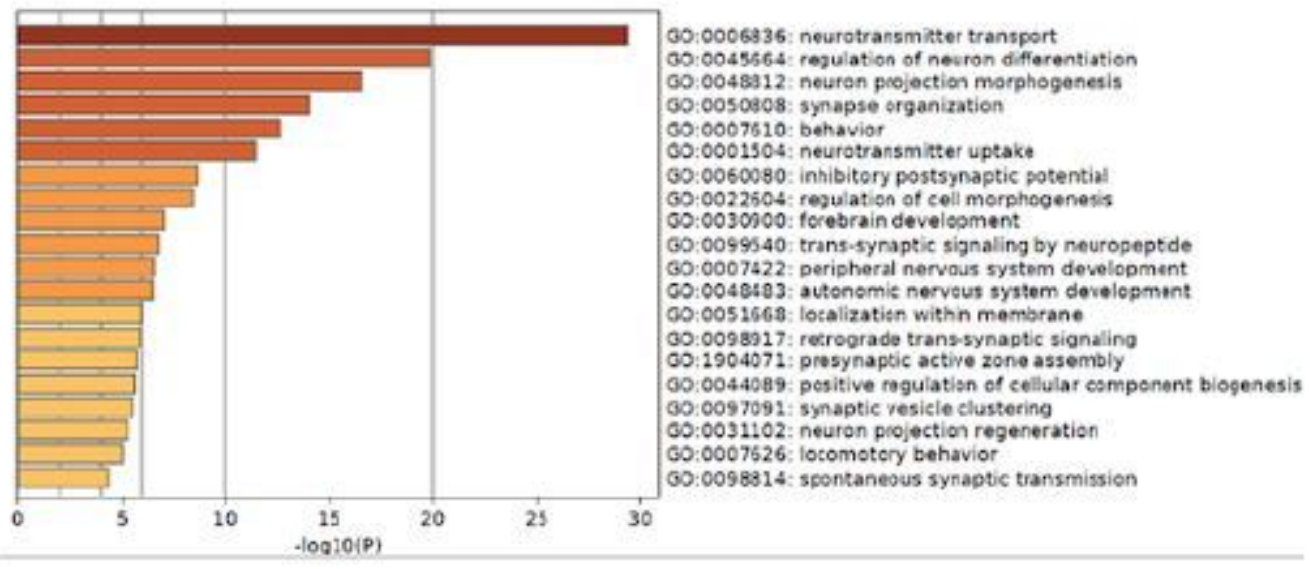

\section{Figure 2}

Heatmap representation of the biological phenomena in which the DE miRNA in SPERM (A), PFC (B) and HIPPO (C) are involved. The colors of the bars indicate the values of $\mathrm{P}$; the darker the colors, the lower the $P$ value. Analysis was performed using rat database. 
3A

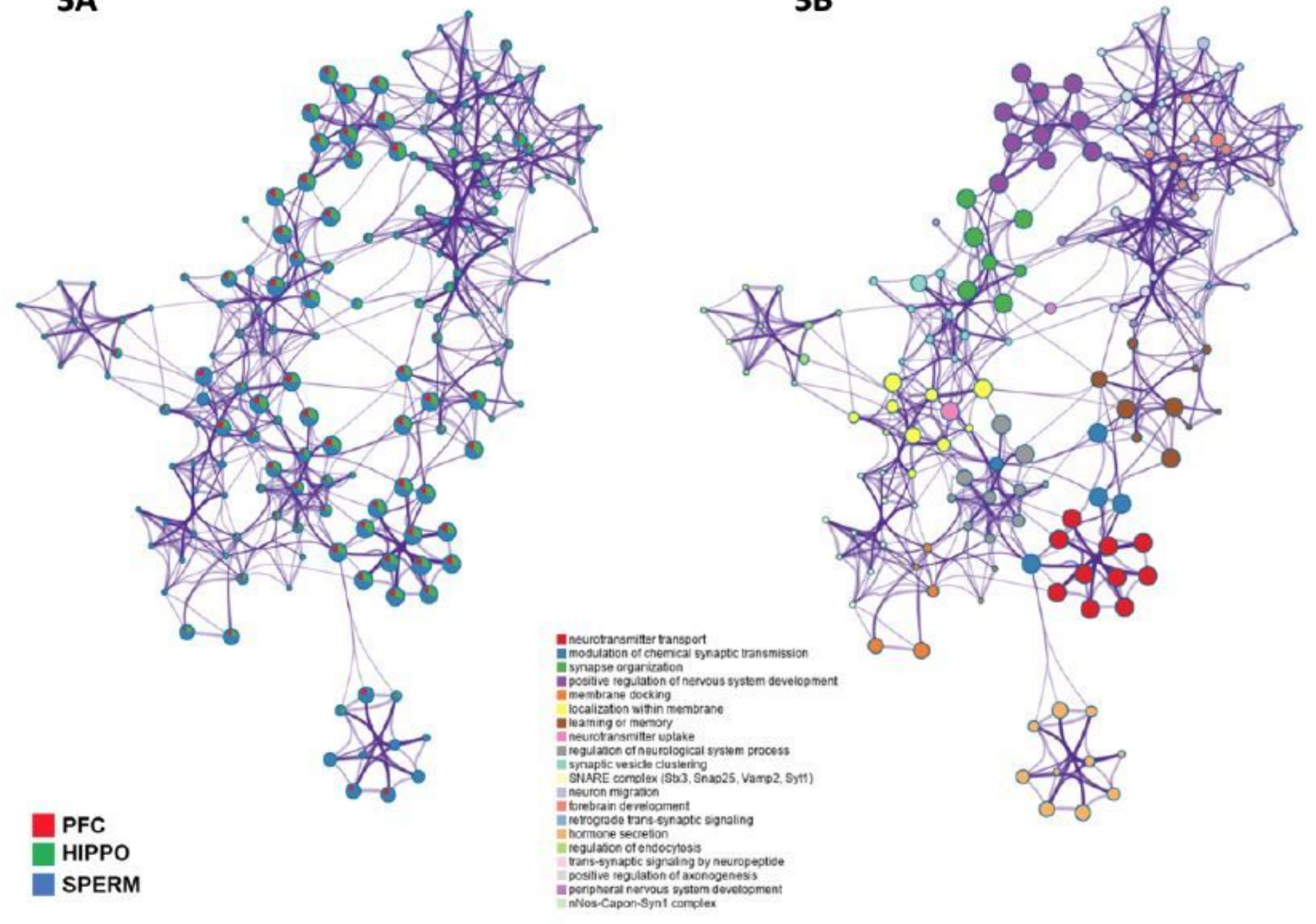

Figure 3

Enrichment network (Gene Ontology Enrichment) in which the nodes represent the hits involved in each biological process. In A the nodes are represented as pie charts. Each portion of the pie represents a sample, and the size of each portion represents the proportion of each hit in each sample. Each hit includes the genes involved in biological processes, which are identified by the colors in B. The size of the nodes reproduces the amount of genes involved in the hits and the purple lines between the nodes represent the interactions. Analysis using the rat database. 


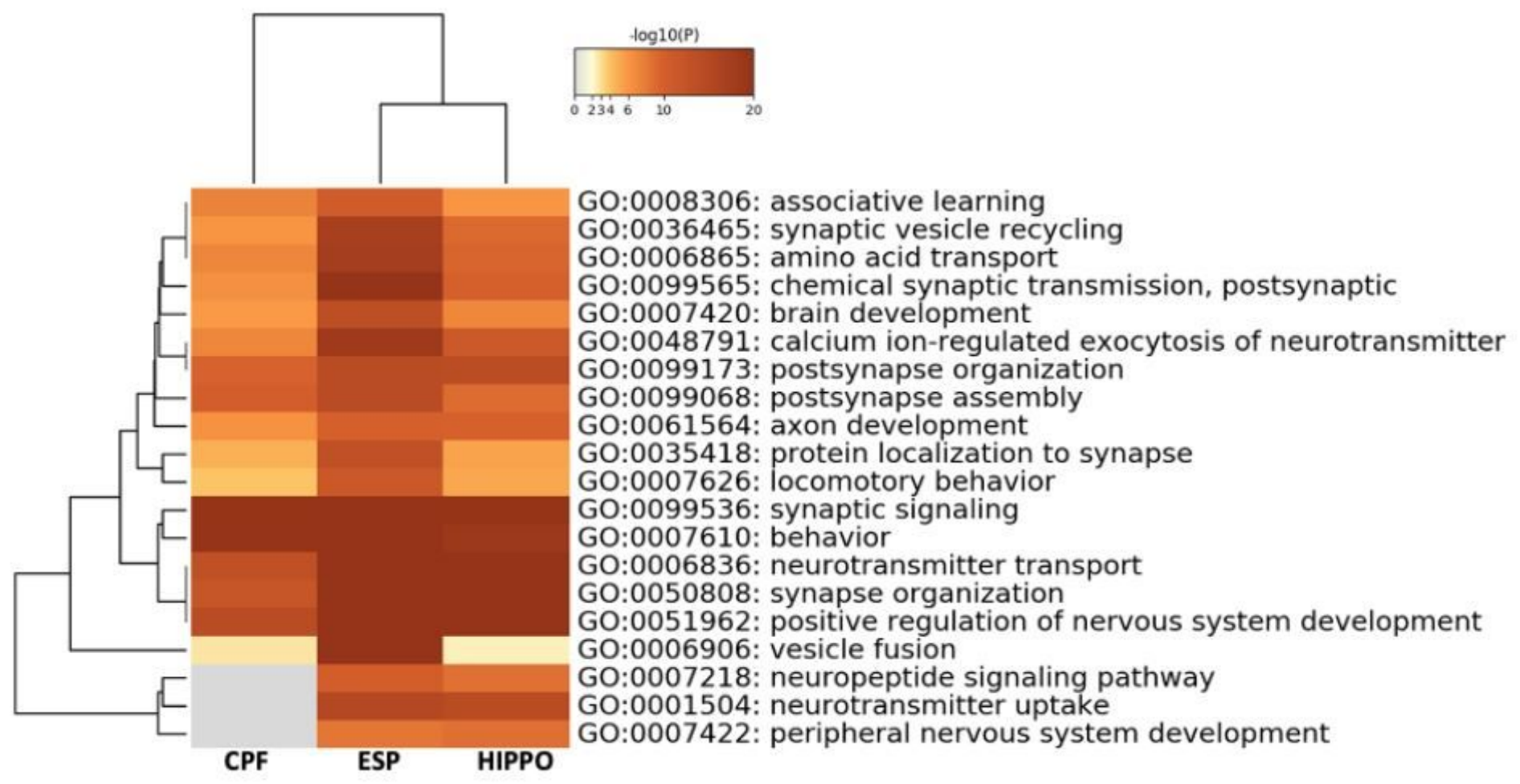

Figure 4

Hierarchical heatmap illustrating the relationship between the identified biological processes and between three types of sample. The colors of the rectangles indicate the $\mathrm{P}$ values; the darker the colors, the lower the value of P. It is observed that there is considerable similarity between the hippocampus and sperm. Synaptic organization and signaling, learning and memory, and neurodevelopment appear as processes that could be significantly affected in the three types of sample. Analysis was performed using the rat database. 


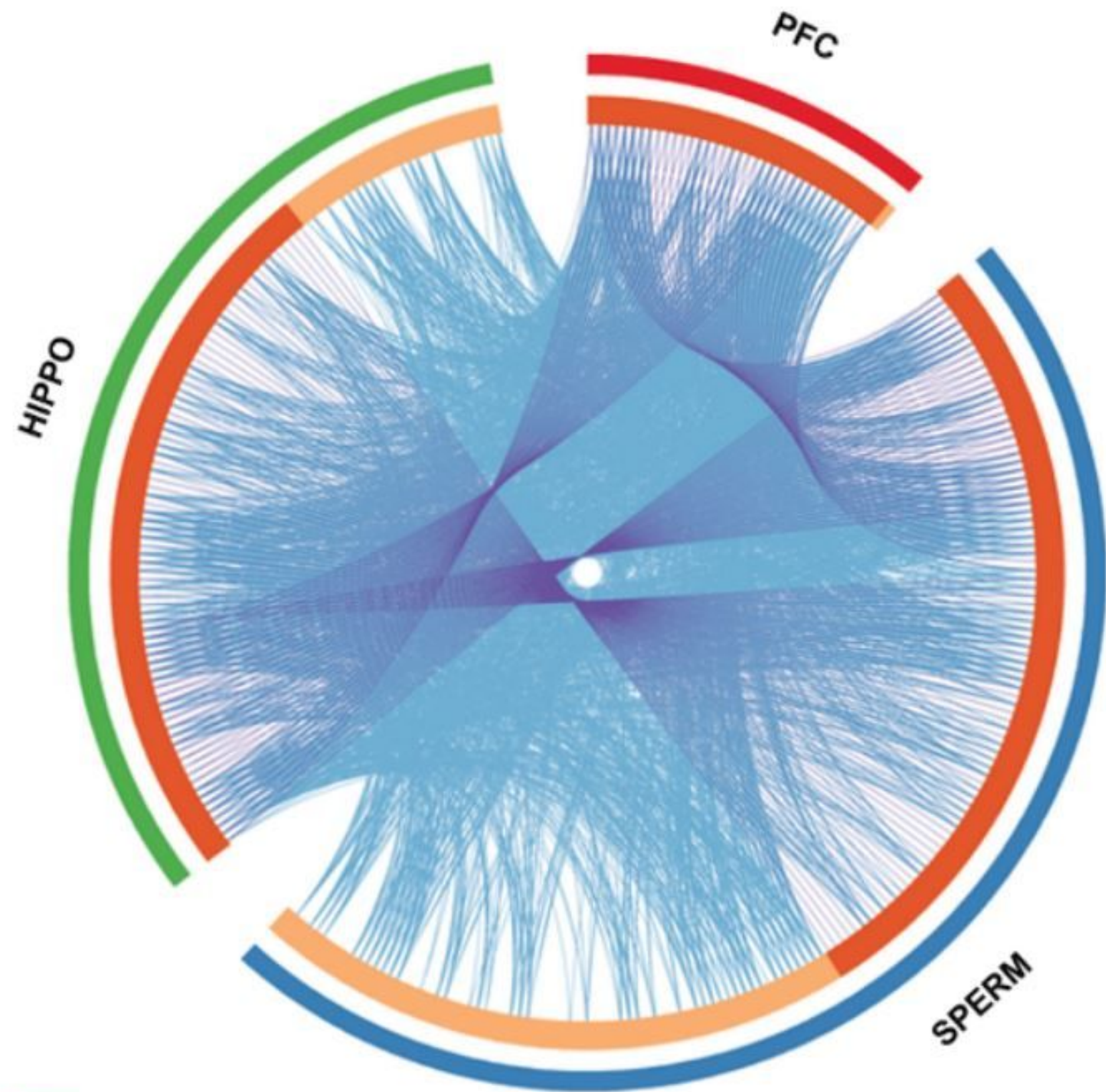

PFC

Sperm

Hippo

Genes that appeas in only one sample

Genes that appeas in more than one sample

\section{Figure 5}

Circos Plot illustrates how differently expressed miRNA target genes coincide between the three types of sample. The outermost arches, represented in blue, red, and green, indicate sperm, prefrontal cortex, and hippocampus, respectively. The internal arcs, represented by the colors dark orange and light orange, represent the genes that appear in more than one type of sample or in a single type of sample, respectively. The purple lines indicate genes that appear in more than one type of sample. The blue lines 
link genes that are in the same "gene ontology term"; in different types of samples. Analysis was performed using rat database.

\section{Supplementary Files}

This is a list of supplementary files associated with this preprint. Click to download.

- SUPPLEMENTARYMATERIAL.pdf

- AppealSUPPLEMENTARYMATERIAL.pdf 